\section{Affiliations}

- E.S. Colizzi (* corresponding author: e.s.colizzi@math.leidenuniv.nl): Mathematical Institute, Leiden University; Origins Center, the Netherlands

- B. van Dijk: Department of Microbial Population Biology, Max Planck Institute for Evolutionary Biology, Plön, Germany

- R.M.H. Merks: Mathematical Institute, Leiden University; Institute of Biology, Leiden University, the Netherlands

- D.E. Rozen: Institute of Biology, Leiden University, the Netherlands

- R.M.A. Vroomans: Informatics Institute, University of Amsterdam; Origins Center, the Netherlands

\title{
Evolution of genome fragility enables microbial division of labor
}

\begin{abstract}
Division of labor can evolve when social groups benefit from the functional specialisation of its members. Recently, a novel means of coordinating division of labor was found in the antibioticproducing bacterium Streptomyces coelicolor, where functionally specialized cells are generated through large-scale genomic re-organisation. Here, we investigate how the evolution of a genome architecture enables such mutation-driven division of labor, using a multi-scale mathematical model of bacterial evolution. We let bacteria compete on the basis of their antibiotic production and growth rate in a spatially structured environment. Bacterial behavior is determined by the structure and composition of their genome, which encodes antibiotics, growth-promoting genes and fragile genomic loci that can induce chromosomal deletions. We find that a genomic organization evolves that partitions growth-promoting genes and antibiotic-coding genes to distinct parts of the genome, separated by fragile genomic loci. Mutations caused by these fragile sites mostly delete growth-promoting genes, generating antibiotic-producing mutants from non-producing (and weakly-producing) progenitors, in agreement with experimental observations. Mutants protect their colony from competitors but are themselves unable to replicate. We further show that this division of labor enhances the local competition between colonies by promoting antibiotic diversity. These results show that genomic organisation can co-evolve with genomic instabilities to enable reproductive division of labor.
\end{abstract}


Motivation of current work Division of labor can evolve if trade-offs are present between different traits. To organize a division of labor, the genome architecture must evolve to enable differentiated cellular phenotypes. Cell differentiation may be coordinated through gene regulation, as occurs during embryonic development. Alternatively, when mutation rates are high, mutations themselves can guide cell and functional differentiation; however, how this evolves and is organized at the genome level remains unclear. Here, using a model of antibiotic-producing bacteria based on multicellular Streptomyces, we show that if antibiotic production trades-off with replication, genome architecture evolves to support a mutation-driven division of labor. These results are consistent with recent experimental observations and may underlie division of labour in many bacterial groups.

\section{Introduction}

Multicellular organisms provide a clear example of a reproductive division of labor, where the germline produces gametes that generate offspring, while the disposable somatic tissues carry out functions that improve survival. Similar divisions of labor are found in colonies of social insects, where one or few individuals are responsible for all of the reproduction, whereas the rest of the individuals perform tasks that mirror those of somatic cells in multicellular organisms. Recently, several striking examples of reproductive and other divisions of labor have been described in the microbial world [1-5]; it has even been proposed that reproductive division of labor existed before the Origin of Life, among prebiotic replicators [6-8]. Thus, such divisions of labor are ancient and ubiquitous, although the mechanisms driving them may be diverse.

In a multicellular reproductive division of labor, somatic cells typically carry the same genetic information as the germline. Cell specialisation is brought about by a combination of gene regulation and epigenetics, ensuring that only a small subset of the genome is expressed. However, an alternative route to reproductive division of labor has been recently proposed in the antibiotic-producing bacterium Streptomyces coelicolor, which generates somatic cells through mutations rather than gene regulation. Here, we propose that the genome of $S$. coelicolor has become structured over evolutionary time such that mutations occur frequently to yield differentiated cells, giving rise to a reproducible division of labour.

Streptomycetes are multicellular bacteria that grow from haploid spores, first producing a so-called vegetative mycelium, and then differentiating into aerial hyphae that produce environmentally resistant spores. During growth, colonies produce a diverse repertoire of secondary metabolites, including antibiotics that are used to regulate competitive interactions between strains. Recent results suggest that antibiotic production and spore 
formation are carried out by distinct cell types. Antibiotic synthesis and secretion are metabolically expensive tasks that trade-off with replication [9]; accordingly, colony fitness is expected to be higher when these tasks are partitioned into separate cells [10].

The antibiotic-hyperproducing subset of cells in $S$. coelicolor colonies arises due to massive and irreversible deletions at the left and right arms of the Streptomyces linear chromosome [9]. Cells with larger deletions produce more antibiotics, but also produce significantly fewer spores, a deficit that effectively ensures their elimination during each replicative cycle [11]. They are instead repeatedly re-generated independently in each colony following spore germination. This process gives rise to heterogeneous colonies containing a diversity of mutants with different chromosome sizes that produce different combinations of antibiotics, as well as a larger fraction of cells specialized for spore production.

The irreversible mutational mechanism used to generate division of labour in S. coelicolor may be widespread in the genus, which is well known for its frequent genome instability [12-15]. But how does this instability reliably generate antibiotic-producing cells? Here, we hypothesize that chromosomal gene order has evolved so that some functional groups of genes are located at the telomeric ends of the chromosome, making them more susceptible to deletion due to genome instability. By this argument, genome instability becomes adaptive within the context of this genome organization, because it facilitates the generation of sterile antibiotic-producing mutants from replicating cells. We show that a genome architecture capable of generating mutation-driven division of labor evolves in a mathematical model of antibiotic-producing bacterial colonies.

\section{Results}

Model overview We formulated a mathematical model based on a simplified description of the multicellular life cycle and ecology of Streptomyces. We focus on the vegetative growth stage, especially during the developmental transition to sporulation [2]. In this phase, colonies grow, interact and compete with one another for space and resources $[13,16,17]$. Secreted antibiotics diffuse around the producing colony, protecting it from competing strains and allowing it to claim the space into which it can grow [18]. Finally, sporulation is induced when colonies experience resource limitation.

We model Streptomyces-like cells that produce antibiotics and replicate on a twodimensional surface. We implicitly account for the metabolic cost of producing antibiotics by incorporating a trade-off between cell division and antibiotic production, which may arise when energy is redirected from primary to secondary metabolism, or due to extensively pleiotropic gene regulation [19-21]. The genome organization of these virtual cells evolves through multiple cycles of vegetative growth and sporulation. 
Each cycle of vegetative growth starts with a population of germinating spores. Bacteria replicate locally, into empty lattice sites in their direct neighborhood. Due to local replication and dispersal, related bacteria remain close to one another and form colonies (Fig.1a). Colonies develop for a fixed number of time steps $\tau_{s}$ (in each time step, all lattice sites are updated in random order) - after which we assume that resources are depleted and colonies sporulate. Sporulation consists of randomly sampling a small fraction $\xi$ of the bacteria, to seed the next cycle of growth and competition. Spores do not disperse between cycles (i.e. there is no spore mixing).

Each bacterium possesses a genome that determines replication rate and antibiotic production. We model the Streptomyces linear genome with a beads-on-a-string model which represents genomes as a linear sequence of genes and other genetic elements [22, 23]. In addition to growth-promoting and antibiotic-production genes, we include fragile genomic sites that are mutational hotspots. These fragile sites can represent, e.g., long inverted repeats or transposable elements, that are common within bacterial genomes $[24,25]$. A genome can contain a variable number of genes and fragile sites.

We specify a direct correspondence between the number of growth-promoting genes $g$ and replication rate $k_{\text {repl }}=R \alpha_{g} g /\left(g+h_{g}\right)$, and - in accordance with experimental results an inverse relationship between growth rate and antibiotic production $k_{\mathrm{ab}}=A \exp \left(-\beta_{g} g\right)$ (plotted in in Fig.1b; $R$ is a cell's resistance to the antibiotics it is in contact with, $\alpha_{g}$ is the maximum replication rate, $h_{g}$ is the number of growth-promoting genes producing half maximum growth rate, $A$ is the maximum antibiotic production rate, $\beta_{g}$ scales the inhibition of antibiotic production with the number of growth-promoting genes). Therefore, the number of growth-promoting genes $g$ trades antibiotic production for replication. This trade-off accounts for the metabolic cost of antibiotic production.

We make the simplifying assumption that the metabolic strategy of each cell, i.e., the amount of resources dedicated to growth vs. antibiotic production, is determined solely by its genotype - and ignore that these strategies may be regulated by density-dependent or other secreted cues from other bacteria [26-28]. Antibiotics produced by a bacterium are secreted into its neighborhood, within a circle of radius $r_{a}=10$ lattice sites. Bacteria can produce different antibiotics, and multiple different antibiotics can be present at each lattice site. The type of an antibiotic is determined by a bit-string of length $\nu$, so therefore the total number of possible antibiotics is $2^{\nu}$. Bacteria are resistant to antibiotics if they encode an antibiotic-production gene with the same bit-string in their genome. Resistance decreases when the difference between the two strings increases. Cells die, and are removed from the lattice, if they come in contact with an antibiotic to which they are not resistant. 


\section{a. Spatially structured ecology of Streptomycetes}

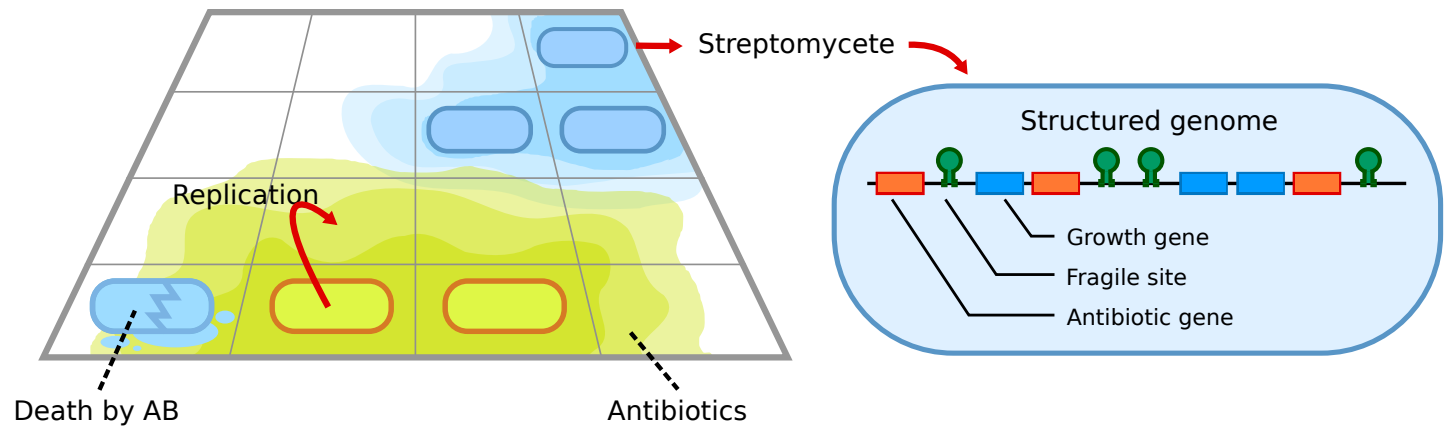

b. Trade-off reproduction vs. AB-production trade-off

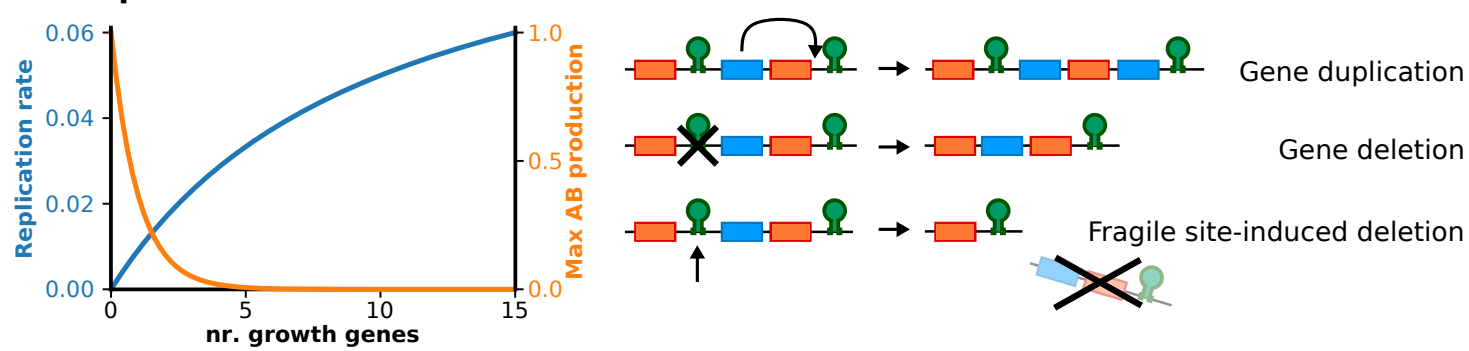

Figure 1: The model: a population of virtual Streptomyces evolving through many cycles of colonial growth of duration $\tau_{s}=2500$ time steps. a Bacteria replicate locally on a two-dimensional surface. They produce antibiotics, which are placed in their vicinity, to which other bacteria may be susceptible. Each bacterium contains a genome - a linear sequence of genes and genetic elements. We consider two gene types - growth-promoting genes and antibiotic genes - as well as fragile sites. $\mathbf{b}$ The metabolic strategy of a bacterium is determined by its genome: a larger number of growth-promoting genes translates to higher growth and lower antibiotic production. Default parameter values are: $a_{g}=0.1, h_{g}=10, \beta_{g}=1$, unless explicitly stated otherwise. c Bacterial genomes mutate during replication: single gene duplications and deletions occur at random locations on the genome with probability $\mu_{d}=10^{-3}$ per gene, whereas large-scale deletions occur at the genomic location of fragile-sites with probability $\mu_{d}=10^{-2}$ per site.

Eco-evolutionary dynamics of virtual Streptomyces Starting from short genomes (initial size $=10$ genes) without fragile sites, containing homogeneously distributed antibi- 
otic genes and growth-promoting genes, we let bacteria evolve over at least 500 vegetative growth cycles, each of $\tau_{s}=2500$ time steps. Over this time, bacteria incorporate many fragile sites in their genome (Fig. 2a), evolve a large and diverse set of antibiotic genes, and a smaller number of growth-promoting genes (see Suppl. Section S1 for additional runs). After evolution, the spatial dynamics over the course of one growth cycle qualitatively reproduce those observed in experiments. Colonies expand and produce a growing halo of antibiotics (see, e.g., pictures in [9]). This expansion continues until colonies encounter antibiotics to which they are susceptible - i.e. antibiotics produced by other colonies. When all colonies are surrounded by antibiotics to which they are susceptible, the invasion dynamics reach a quasi-stable spatial configuration [29] (Fig. 2b; see Suppl. Section S2 for more snapshots, and Suppl. Video 1).

\section{Evolution of genome architecture leads to division of labor between replicating and} antibiotic-producing bacteria To understand the population dynamics produced by the model, we compare populations after different stages in the simulations. At an early stage (after 40 growth cycles, i.e. $100 \times 10^{3}$ time steps), antibiotics are collectively produced by the whole population. By contrast, at a later stage ( 400 cycles, i.e., $1000 \times 10^{3}$ time steps) nearly all antibiotics are produced by a small fraction of the bacteria (Fig. 2c, 99\% of all antibiotics are produced by $61 \%$ of the population at time $100 \times 10^{3}$, and by just $14 \%$ at time $1000 \times 10^{3}$ ). We also observe that replication and antibiotic production are performed by genetically distinct bacteria in the later stages: bacteria with few growth-promoting genes produce most antibiotics but do not replicate frequently, whereas bacteria with a larger number of growth-promoting genes replicate frequently but do not produce antibiotics (Fig. 2d). Because antibiotic-producing bacteria do not replicate autonomously, they cannot form an independent lineage, and are instead generated in each growth cycle from the bacteria that have high replication rates. A strong trade-off between replication and antibiotic production is required for this division of labor to emerge (Suppl. Section S3).

Genome architecture supports the mutation-driven division of labor To understand the role of genome architecture in the division of labor, we extracted the founder of the most abundant colony after long-term evolution, and tracked its diversification after it was reinoculated into an empty grid (for ease of interpretation the only mutations we allow in this experiment are fragile site-induced deletions). We observed that the genome of the evolved colony founder had two distinct regions: a telomeric region that contains growth-promoting genes but lacks fragile sites, and a centromeric region that lacks growth-promoting genes and has abundant fragile sites (Fig. 3). After colony growth from this founder, nearly all the bacteria capable of replicating are genetic copies of the founder, while most of the antibiotic production is carried out by a diverse suite of mutants arising through genomic instabilities (Fig. 3a). When bacteria divide, mutations induced at fragile sites lead to the deletion of the part of the genome distal to them, causing large 


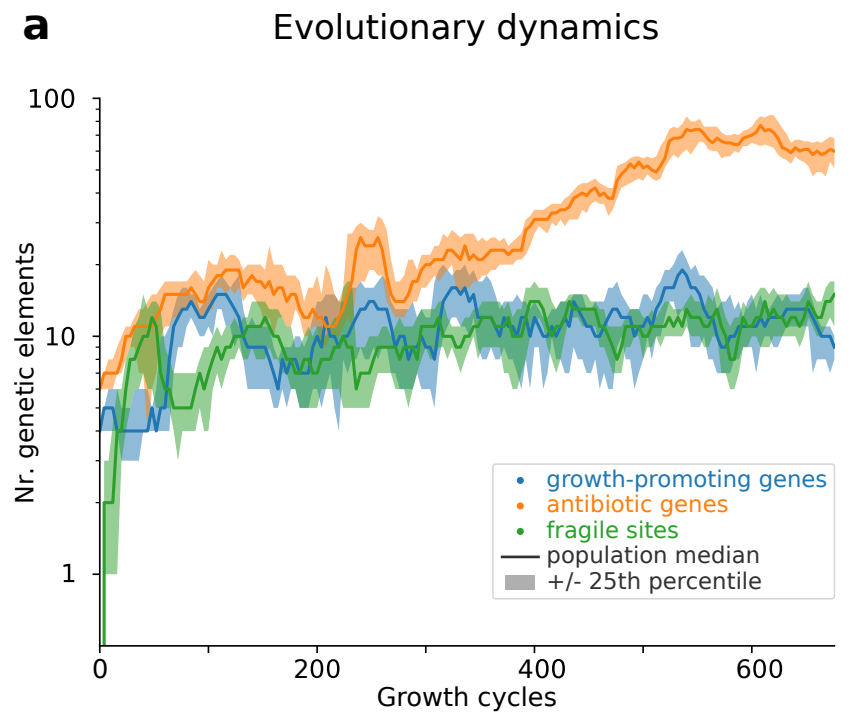

b Spatial dynamics (snapshots)

over one growth cycle

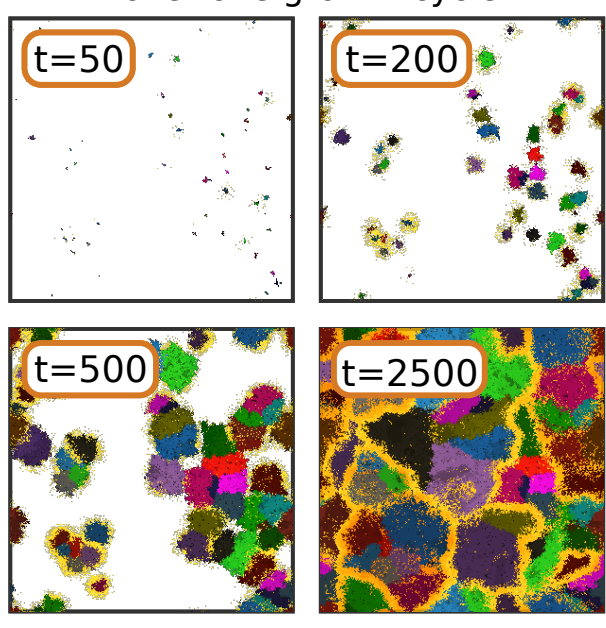

C Antibiotic production in the population
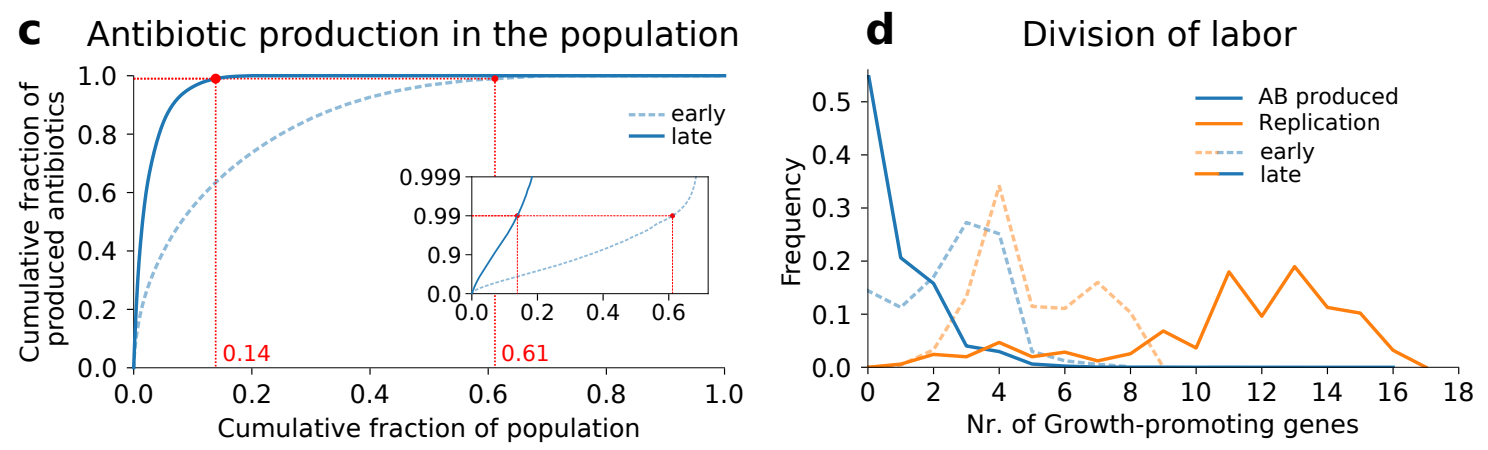

Figure 2: Evolution of genome composition and division of labor. a Evolutionary dynamics of gene content. We collect the genomes of all bacteria at the end of the growth cycle (every 10 cycles), and we count the number of genes subdivided by type in each genome. The plot shows the median and 25 th percentile deviation. Growth cycle duration $\tau_{s}=2500$ time steps. Notice the logarithmic y-axis. b Spatial dynamics during one growth cycle, after evolution: different colors represent different colonies, yellow shading around the colonies indicates antibiotics, time stamps in the pictures indicate the time elapsed in the growth cycle. c Most antibiotics are produced by few bacteria after evolution. We collect all bacteria alive at the midpoint of the growth cycle of an early (after 40 cycles, i.e. $100 \times 10^{3}$ time steps) and later stage (after 400 cycles, i.e. $1000 \times 10^{3}$ time steps) in the evolutionary dynamics (i.e. at 1250 time steps). For each time point, bacteria are sorted on antibiotic production, the cumulative plot (blue line) is normalized by population size and total amount of antibiotics produced, the red dot indicates the fraction of the population that produces $99 \%$ of all antibiotics. Inset: semi-log plot of the same data. $\mathbf{d}$ Division of labor between replication and antibiotic production, shown as a function of the number of growth-promoting genes. Using the same data set as $\mathbf{c}$, the plot shows the frequency of replication events (orange) and antibiotic production events (blue) per number of growth genes, for early and later stage in evolution. 
telomeric deletions. Because growth-promoting genes are over-represented in these regions, they are frequently deleted as a group (Fig. 3c). Mutants generated from these deletions lack growth-promoting genes while retaining many antibiotic genes, and will therefore produce antibiotics at much higher rates (Fig. 3d). By this process, colonies that begin clonally evolve to become functionally differentiated, and contain throughout the growth cycle on average $2 \%$ to $7 \%$ mutants with large telomeric deletions that specialize in antibiotic production while foregoing replication themselves (Suppl. Section S4). In Supplementary Section S5 we show this genome architecture is prevalent in the evolved population.

To assess whether genome architecture is required for division of labor, we initiated an evolutionary run where we shuffled the order of the genes in the genome of each spore at the beginning of every growth cycle. This disrupts the genomic architecture of colony founders without changing its composition, in terms of the number and type of genes and fragile sites. Starting from an evolved genome, we observe a rapid decrease of antibiotic genes and, surprisingly, of growth-promoting genes, resulting in small genomes (Fig.4a). At evolutionary steady state, a large fraction of the population contributes to antibiotic production (Fig.4b). These bacteria do not divide labor, because they can both replicate and produce antibiotics (Fig.4c), but at a lower rate overall than when these tasks are divided. Thus they evolve a genome that does not resolve the trade-off, but compromises between replication and antibiotic production. This confirms that the structure of the genome is an important prerequisite for the maintenance of a mutation-driven division of labor.

\section{Effect of spatial competition dynamics and antibiotic diversity on evolution of di-} vision of labor We next examined the effect of spatial structure on the evolution of division of labor in our model. Previous work showed that spatial structure can promote the coexistence and diversity of antibiotic producing cells, because antibiotics secreted in a cell's neighbourhood prevent invasion by adjacent strains [16, 29-32]. In our model, antibiotic-based competition occurs once the boundaries of two colonies come into contact. This requires sufficiently long growth cycles ( $\geq 1000$ time steps; see Suppl. Section S6). No division of labor evolves when cycles are shorter because selection favors only growth, and growth cycles shorter than 500 time steps lead to extinction as populations do not recover after sporulation. Furthermore, spatial structure can be destroyed entirely by mixing the system every time step. We find that evolved bacteria maintain mutationdriven division of labor in such a mixed system (Suppl. Section S7, Fig. SF8), but division of labor cannot evolve de novo from random genomes (Fig. SF9).

In line with previous work [29], we find that colonies produce a large number of different antibiotics, which results from selection for diversity (Suppl. Section S8). When the 


\section{a}

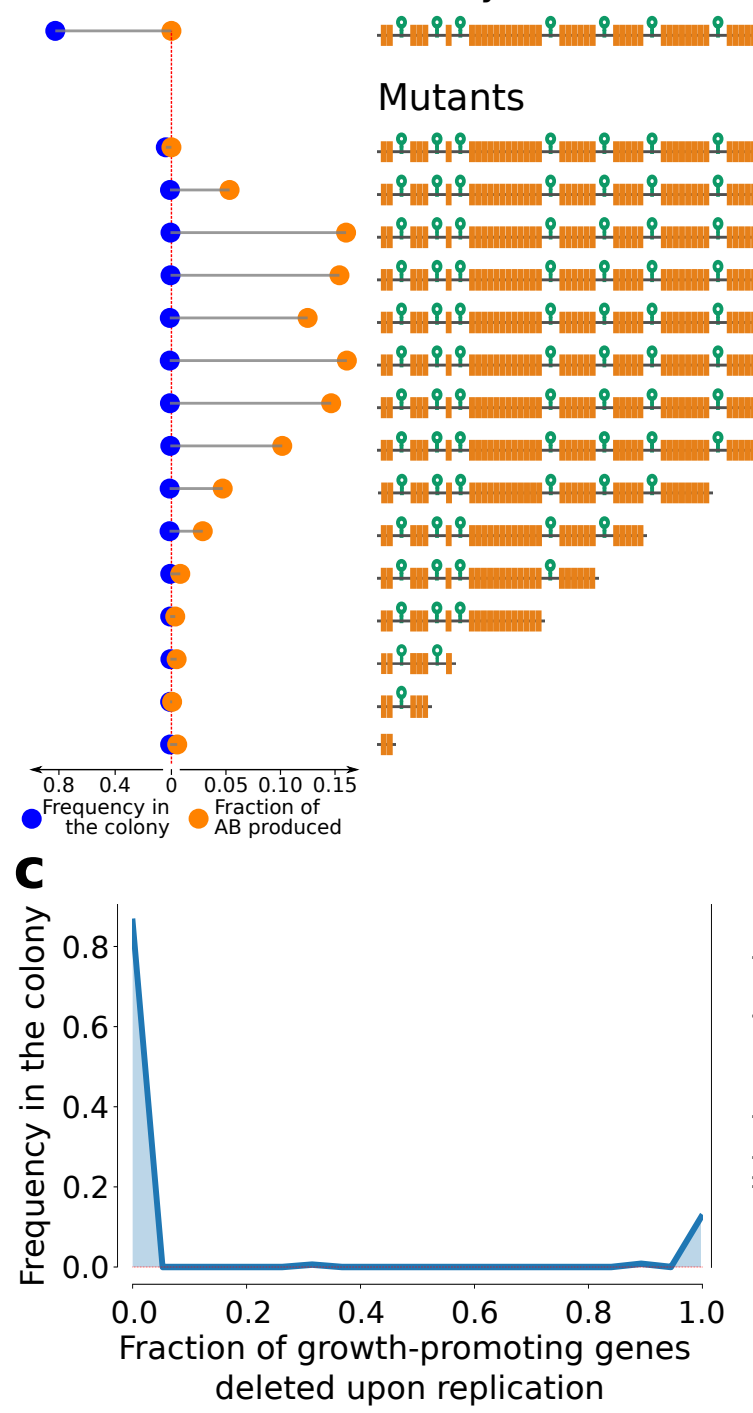

b

\section{Colony founder}

\section{Mutants}
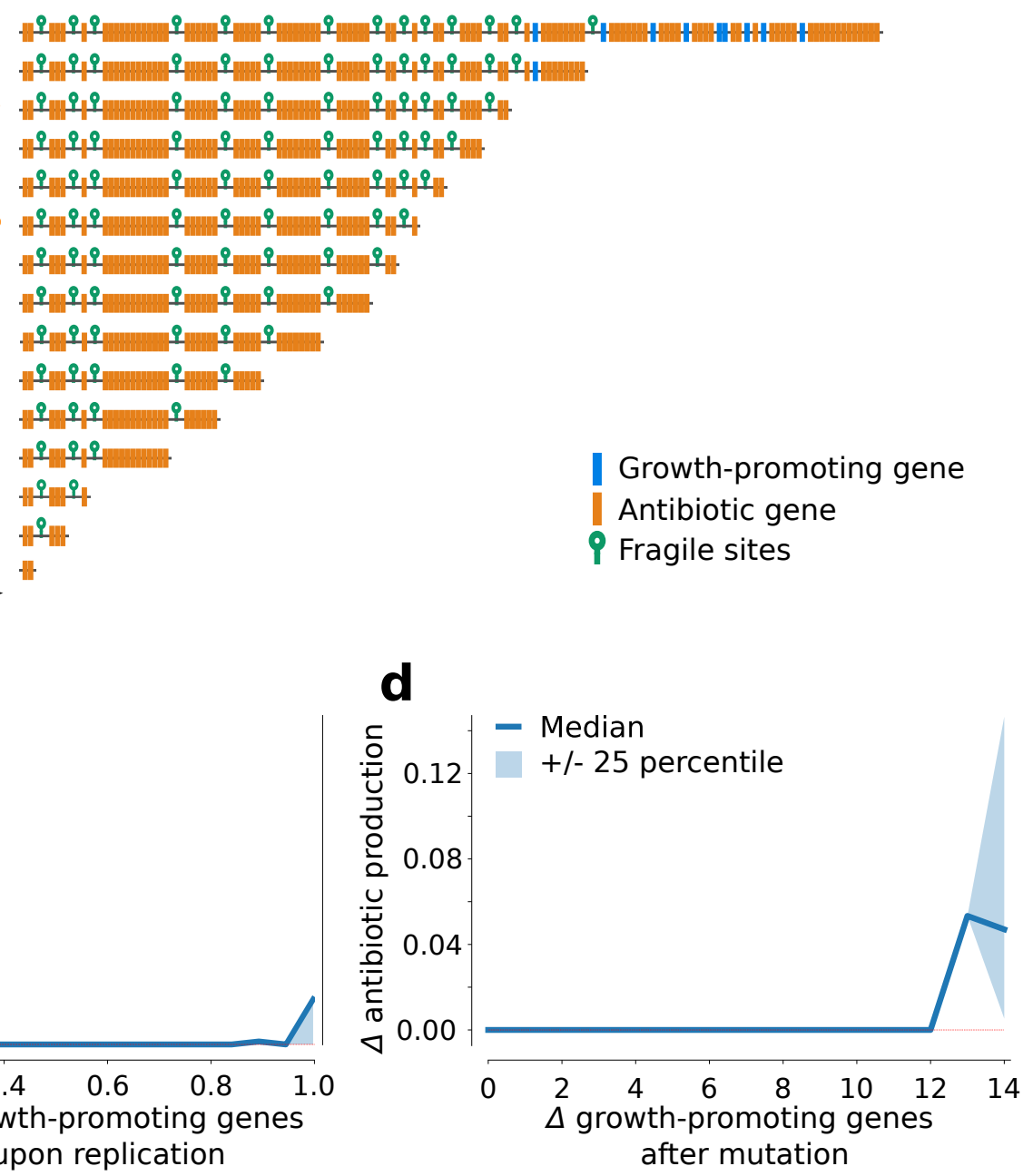

Figure 3: Division of labor within an evolved colony: the colony founder generates antibioticproducing somatic cells through large-scale chromosomal deletions, caused by fragile sites. The data is generated by seeding a simulation with one bacterium - the colony founder - and letting the colony grow until it reaches a diameter of 70 lattice sites (which is the approximate colony size at the end of a growth cycle, cf. Fig. 2b). a Genomes in high abundance do not produce many antibiotics. Bacterial genomes are collected and sorted by abundance in the colony. The bar-plots contrast genome frequency in the colony (blue) and the fraction of antibiotics produced by bacteria with that genome (orange). b Genome architecture of the colony founder and all its descendants. Growth-promoting genes in blue, antibiotic genes orange, fragile sites depicted as green hairpins. c Drastic and reliable deletion of growth-promoting genes after replication. We recorded the fraction of growth-promoting genes deleted because of fragile site instability after each replication event during colony development. The plot shows the frequency of deletion sizes (between 0 , i.e. no genes are deleted, and 1 - all growth genes are deleted). d Chromosomal deletions induce antibiotic production. Difference in antibiotic production as a function of the growth-promoting genes lost after replication (colony median $+/-25$ th percentile). 


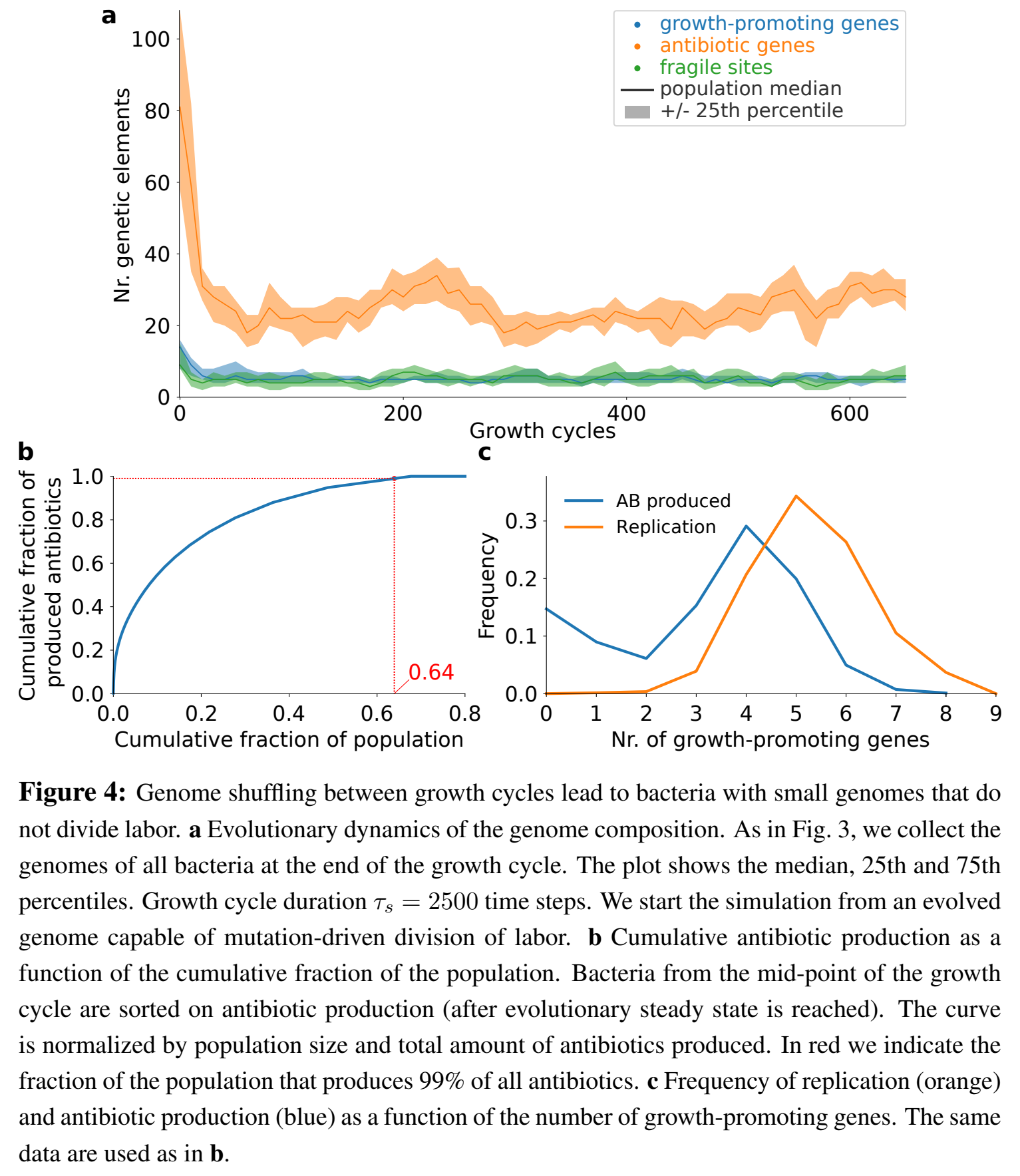


total number of possible antibiotics is large (about $6.5 \times 10^{5}$ different antibiotics, see Methods), colonies are highly susceptible to the antibiotics produced by other colonies (Suppl. Section S9). With a small number of possible antibiotics, bacterial genomes evolve to contain every possible antibiotic gene, which results in low susceptibility (Suppl. Section S10, also cf. [30]). Nevertheless, mutation-driven division of labor evolves in both cases (Suppl. Section S11).

\section{Discussion}

We studied how an association between genome architecture and division of labor evolves, inspired by recent experimental findings in Streptomyces [9]. We constructed a mathematical model of Streptomyces multicellular development where cells have a linear genome with mutational hotspots, and experience a trade-off between replication and antibiotic production. We showed that replicating bacteria can differentiate, via mutation, into a cell type that specializes on antibiotic production, thereby resolving the trade-off between these traits (Fig. 2). Differentiation is coordinated by an evolved genome architecture with two key features: fragile sites that destabilize the genome by causing large-scale deletions, and an over-representation of growth-promoting genes at the unstable chromosome ends. Because of this organization, mutations at fragile sites preferentially delete growth-promoting genes, leaving mutant genomes containing only genes for antibiotic production. Although these cells are unable to grow, their terminal differentiation into antibiotic-producing cells is advantageous to the colony as a whole.

It is well known that Streptomyces genomes contain a conserved core region, while the terminal regions of the chromosome show inter- and intra-specific variation [33]. Our model suggests that telomeric instabilities have evolved to coordinate the division of labor between replication and antibiotic production, explaining recent experimental results in S. coelicolor [9]. Therefore, these instabilities may not be accidental byproducts of a linear chromosome or other aspects of unstable replication [12], but are instead an evolved and functional property of Streptomyces genomes. Based on the robustness of our results, and because genome instabilities are extremely common in Streptomyces, we predict that other species in the genus also divide labor through this mechanism. Streptomyces produce a broad diversity of metabolically expensive compounds like cellulases and chitinases that can also be used as public goods, which are also expected to trade-off with growth. Divisions of labor that arise due to genome instability may therefore not be specific to just antibiotics and reproduction. These divisions of labor could alternatively be organized through differential gene expression - as is common in multicellular eukaryotes, eusocial insects and other microbes. However, an advantage of the mutation-driven division of labor presented here is that it makes social conflicts [34] impossible, because altruistic somatic cells do not possess the genetic means to reproduce autonomously and 
participate in social dynamics [35]. Validating these predictions will require detailed experiments in other species, as well as bioinformatic analyses of Streptomyces genome structures. These analyses may then inform a more detailed model of Streptomyces division of labor, accounting for the interplay between genome organization, gene regulation and the metabolic network, underpinning the trade-off between growth and antibiotic production.

Mathematical models indicate that the organization of genetic information along the chromosome is influenced by the mutational operators that act on it [22, 36-39]. As a consequence of this organization, mutations may be more likely to generate mutant offspring with specific characteristics, such as reduced competition with the wildtype due to low fitness [40-43], lower propensity for social conflicts [44] or accelerated re-adaptation to variable environments [45]. In prokaryotes, mutational operators that can drive functional mutagenesis include horizontal gene transfer, which drives the rapid evolution of gene content [31, 46-48], and the CRISPR-Cas system, that generates immunity to viral infections through targeted incorporation of viral genomes [49]. In an Origin of Life model, it was found that mutants could provide a benefit to the wildtype. There a germline RNA replicator evolved whose mutants were sterile but altruistically replicated the germline and protected it from parasites. [6]. Building on these earlier results, our model shows that a genome architecture can evolve to incorporate mutational hotspots, thus exploiting mutations to generate functional phenotypes and divide labor.

Beyond Streptomyces, mutation-driven division of labor occurs in the genome of many ciliates, where functional genes must be carefully excised from a transposon-riddled genomic background before being transcribed $[50,51]$. Programmed DNA elimination in somatic cells is common in multicellular eukaryotes [52], and targeted recombination is essential for the functioning of the adaptive immune system in vertebrates [53]. These examples highlight the ubiquity of functional mutagenesis across the tree of life. Our model may therefore help understand the evolutionary origin of division of labor through functional mutagenesis more broadly.

Conclusions Our model shows that a bacterial colony can divide labor between replication and antibiotic production by evolving a genome architecture that physically segregates genes for these different functions, and which allows them to be dissociated due to mutations at fragile sites. This gives rise to an effective germ-soma division, whereby one class of cells specializes on reproduction, while the other class focuses on costly antibiotic production. In this system, which replicates dynamics observed in $S$. coelicolor, somatic cells enhance colony fitness, despite being sterile. 


\section{Methods}

The model is a lattice-based stochastic simulation system. We consider a population of bacteria that can replicate and produce antibiotics. The model is inspired by the life cycle of Streptomyces coelicolor, and focuses on the hyphal growth phase, when colonies develop and compete by producing antibiotics [2]. We therefore model the eco-evolutionary dynamics occurring during several colony growth cycles, each of fixed duration $\tau_{s}$. At the beginning of each cycle, spores germinate and colonies begin to form through bacterial replication. If antibiotics are produced, they are deposited around the producing bacterium, forming a halo that protects the colony and "reserves" space to replicate into. Bacteria die if they come into contact with antibiotics to which they are sensitive, and therefore bacteria of a colony cannot invade into the antibiotic halo of another one - if they are genetically different. At the end of the cycle, corresponding to the sporulation phase in Streptomyces, a small random sample $\xi$ of the population is selected to form spores. These spores seed the next cycle, and other bacteria and antibiotics are removed from the lattice. Spores are deposited at the same location where the bacterium lived (we do not shuffle the location of the spores), unless explicitly stated.

We model bacteria and antibiotics on two separate lattices $\Lambda_{1}$ and $\Lambda_{2}$. Both lattices have size $L \times L$ and toroidal boundaries to avoid edge effects. Every site of $\Lambda_{1}$ can either be occupied by one bacterium, or be empty. Every site of $\Lambda_{2}$ can be occupied by multiple antibiotics. Each bacterium possesses a genome which determines their replication rate, as well as the rate and type of antibiotics it produces and is resistant to. The genome consists of a linear sequence of three types of genetic elements (so called beads on a string model $[22,23])$. We consider two gene types - growth-promoting genes and antibiotic genes. The third type of genomic element is a fragile genomic site - a hotspot for recombination. Growth-promoting genes increase replication rate and inhibit antibiotic production, in accordance with Streptomyces growth being favored over secondary metabolism. Antibiotic genes encode both the toxin and its resistance ([54]). Antibiotic type is encoded in the genetic sequence of the antibiotic gene. This sequence is modelled as a bit-string of fixed length $\nu$, which can be mutated to encode different antibiotics. Each bacterium can have multiple growth-promoting and antibiotic genes, as well as multiple fragile sites. 


\section{Replication}

Replication rate depends on the intrinsic replication rate function $G$ and on the resistance $R$ of the bacterium to antibiotics in the spatial location in which it lives:

$$
k_{\text {replication }}=G(g) R(\text { antibiotics }) \text {. }
$$

The intrinsic replication rate $G(g)$ is an increasing, and saturating, function of the number of growth-promoting genes $g$ :

$$
G(g)=\alpha_{g} \frac{g}{g+h_{g}},
$$

with $\alpha_{g}$ the maximum replication rate, and $h_{g}$ the number of growth-promoting genes producing half maximum replication rate. Antibiotic resistance $R$ depends on whether a genome has at least an antibiotic gene sufficiently similar to the antibiotics present on $\Lambda_{2}$ at the corresponding location of the bacterium, where similarity is determined from the bit-strings of the antibiotics and the antibiotic genes. For each antibiotic $a$ on $\Lambda_{2}$, the Hamming Distance $D$ (the number of different bits) between the antibiotic and the gene with the minimum distance from $a$ is calculated. All these minimum distances are summed into a susceptibility score $S=\sum D$, and the overall resistance is a decreasing function of $S$ :

$$
R=e^{-\beta_{r} S^{2}}
$$

Each bacterium is resistant to the antibiotics it produces, because for each antibiotic $D=0$, and thus $R=1$. Moreover, the Gaussian function ensures that small mutations in antibiotic types do not decrease resistance too rapidly. Replication occurs when bacteria are adjacent to an empty site on $\Lambda_{1}$. The probability of replication for each competing bacterium $i$, is $k_{\text {replication }}(i) / \eta$, with $\eta$ the neighborhood size (we use the Moore neighborhood throughout this study, thus $\eta=8$ ). Upon replication, the new bacterium inherits the genome of its parent, with possible mutations.

\section{Mutations}

Mutations occur during replication, and can expand or shrink genomes, and diversify antibiotics.

Duplications and deletions Duplication and deletion of genes and fragile sites occur with equal per-gene probability $\mu_{d}$. When a gene or a fragile site is duplicated, the gene copy is inserted at a random genomic location. This ensures that gene clustering is not a trivial consequence of neutral mutational dynamics, and must instead be selected upon to evolve. 
Fragile site large chromosome deletions Fragile sites are the cause of genome instability in the model. We let fragile sites cause large-scale chromosomal mutations with a per-fragile site probability $\mu_{f}$. We take into account that large-scale mutations in Streptomyces preferentially disrupt telomeric regions [12-15] by letting fragile site-induced mutations delete the entire telomeric region offstream of the genomic location of the fragile site (see Fig. 1c).

Mutations of the antibiotic genes The antibiotic genes consist of a genetic sequence modelled as a bit-string of length $\nu$. Mutations flip bits with a uniform per bit (per antibiotic gene) probability $\mu_{a}$. This changes the antibiotic type, and thus the antibiotic repertoire of the bacterium.

Influx of new fragile sites Fragile genomic sites, such as inverted repeats or transposable elements are common in the genome of Streptomyces. Because they are easily copied (or translocated) we assume that they can also be spontaneously generated with a small probability $\mu_{n}$ (independent of genome size). The new fragile site is inserted at a random location in the genome.

\section{Antibiotic production, in a trade-off with replication}

Antibiotic production rate is modelled as an increasing function $A$ of the number of antibiotic genes $a$ a bacterium has. At the same time, production is strongly inhibited by growthpromoting genes - a function $I(g)$ with $g$ the number of growth-promoting genes in the genome, in accordance with a likely trade-off between growth and secondary metabolism [9]. Antibiotic production rate, per time step, is the function:

$$
k_{\mathrm{ab} \text { production }}=A(a) I(g)
$$

with

$$
\begin{aligned}
& A(a)=\alpha_{a} \frac{a}{a+h_{a}}, \\
& I(g)=\exp \left(-\beta_{g} g\right) .
\end{aligned}
$$

According to this function, the trade-off becomes rapidly steeper with larger $\beta_{g}$. For simplicity, we assume that antibiotics are deposited at a random location within a circle of radius $r_{a}$ around the producing bacterium. Moreover, we do not take into account concentrations of antibiotics and only model presence/absence of an antibiotic type in a spatial location. The probability of producing an antibiotic, per site within the circle, per

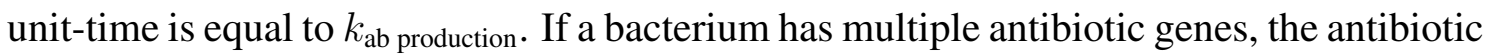
deposited is chosen randomly and uniformly among them. 


\section{Death}

Death can occur when a bacterium is sensitive to an antibiotic located at the same site as the bacterium itself. The probability of a bacterium dying is calculated as $1-R$, where $R$ is the bacterial resistance to the antibiotic defined above. When a bacterium dies, it is removed from the lattice, leaving behind an empty site.

\section{Movement}

Bacteria have a small probability $p_{\text {mov }}$ of moving, if there is an empty site adjacent to them. This speeds up colony expansion and competition between colonies, and avoid strong grid effects that could make spatial patterns too rigid.

\section{Initial conditions and updating of the dynamics}

Unless differently specified, at time $t=0$ a small population of spores is seeded on the lattice. The initial spores have a small genome of length 10, i.e. a random sequence of growth-promoting genes and antibiotic genes (of random types), but no fragile sites. The lattice is updated asynchronously: over one time step, each lattice site is updated in random order.

The source code is written in c, and uses the CASH libraries [55]. Analysis and plotting custom software is written in python. Genome schematics in Fig. 3b are partly drawn with dnaplotlib [56]. The source code and analysis scripts are available at [57]. 
Table 1: Parameters

\begin{tabular}{|c|c|c|}
\hline Parameter & explanation & Values \\
\hline$L^{2}$ & lattice size & $300 \times 300$ \\
\hline$\eta$ & neighborhood size for replication & 8 (Moore neigh.) \\
\hline$\tau_{s}$ & Growth cycle duration & 2500 time steps \\
\hline$\xi$ & Fraction of spores to seed a growth cycle & 0.001 \\
\hline \multicolumn{3}{|l|}{ Replication } \\
\hline$\alpha_{g}$ & max replication probability per unit time & 0.1 \\
\hline$h_{g}$ & nr. of growth genes for $1 / 2 \max$ growth rate & 10 \\
\hline$\beta_{r}$ & antibiotic resistance factor & 0.3 \\
\hline$\mu_{d}$ & Duplication/deletion probability per gene & 0.001 \\
\hline$\mu_{f}$ & Fragile site-induced deletion probability & 0.01 (per fragile site) \\
\hline$\mu_{n}$ & Probability of new fragile site formation & 0.01 per genome \\
\hline$\mu_{a}$ & Probability of antibiotic type mutation & 0.005 (per ab gene) \\
\hline \multicolumn{3}{|c|}{ Antibiotic production } \\
\hline$\alpha_{a}$ & max antib. production probability per unit time & 1 \\
\hline$h_{g}$ & nr. of antib. genes for $1 / 2$ max production rate & 3 \\
\hline$r_{a}$ & max distance of antib. placement & 10 \\
\hline$\beta_{g}$ & antib. production decrease due to trade-off & 1 \\
\hline$\mu$ & length of antib. bit-string & 16 \\
\hline \multicolumn{3}{|c|}{ Other parameters } \\
\hline$p_{\text {mov }}$ & prob. of migration into empty adjacent site & 0.01 \\
\hline
\end{tabular}




\section{Supplementary Material of:}

\section{Evolution of genome fragility enables microbial division of labor}

\section{S1 The evolutionary dynamics of genome composition}

For clarity, in the main text we showed only one example of the evolutionary dynamics. Fig. SF1 shows that qualitatively the same evolutionary trends are followed when the experiment is repeated.

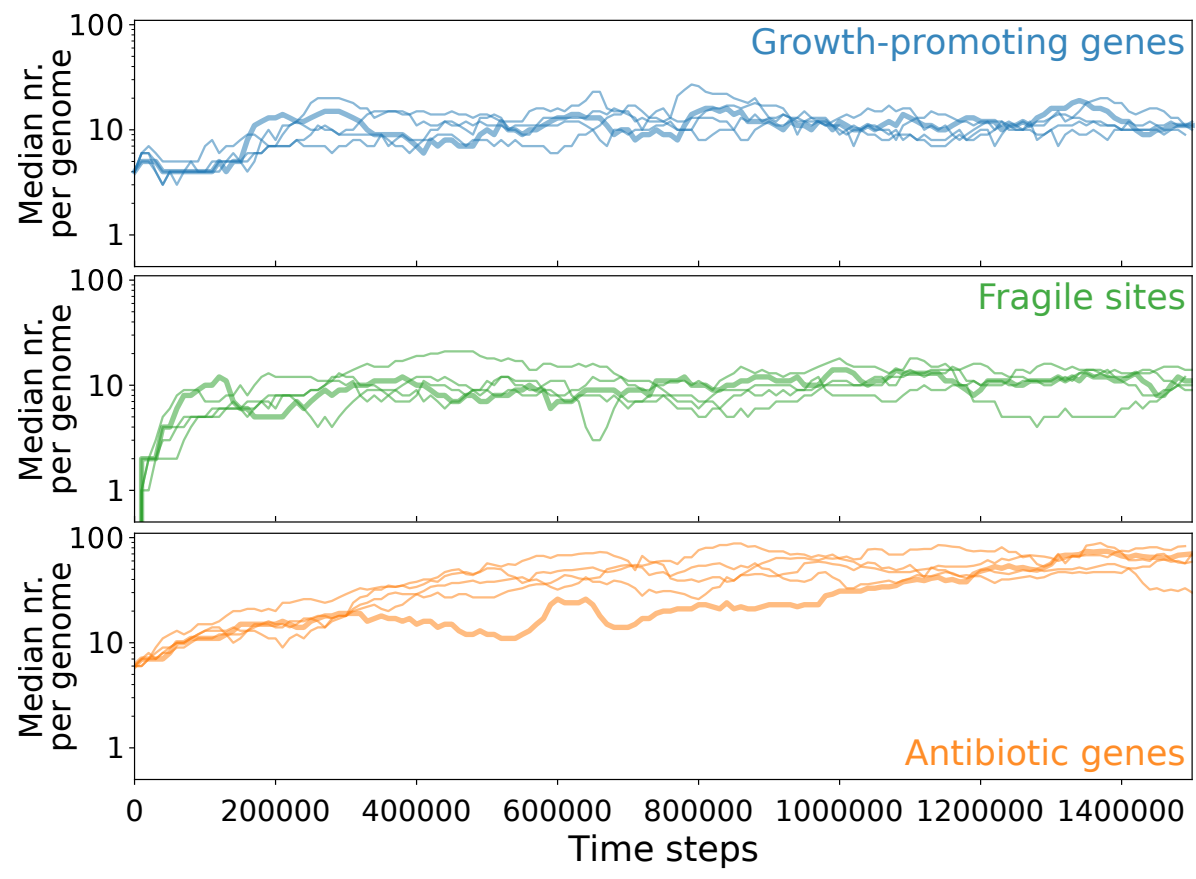

Figure SF1: Evolutionary dynamics of gene content, in five independent evolutionary runs. The run used in the main text is indicated with a thicker line.

The mutation-driven division of labor described in the main text is robustly driving the eco-evolutionary dynamics also when we run the system for a very long time (2000 growth cycles), see Fig. SF2. Interestingly, we also observe large fluctuations in the number of antibiotic genes. 


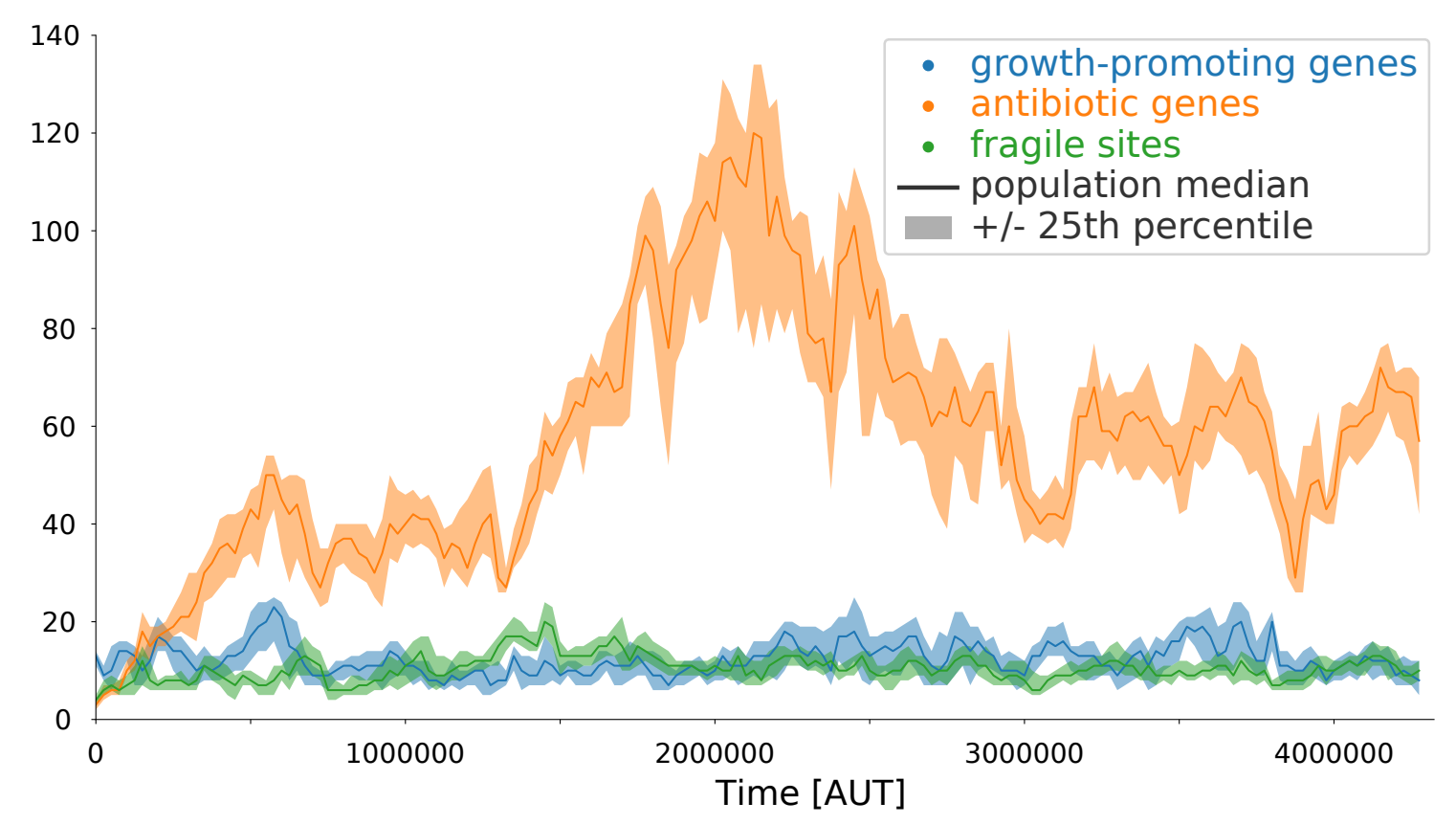

Figure SF2: Very long-term evolutionary dynamics of genome composition. All parameters are the same as in Fig. 2a.

\section{S2 Snapshot of the eco-evolutionary dynamics within one growth cycle}

Fig. SF3 shows successive snapshots of the lattice, over the course of a growth cycle that lasts 2500 time steps. Starting from spores, colonies expand and produce antibiotics. 
Time: 0

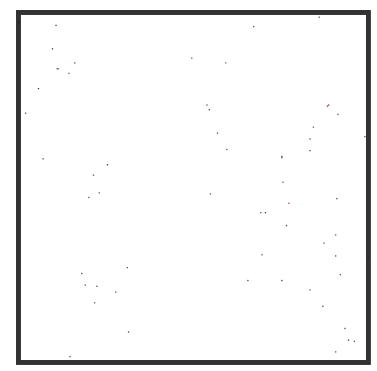

\section{0}

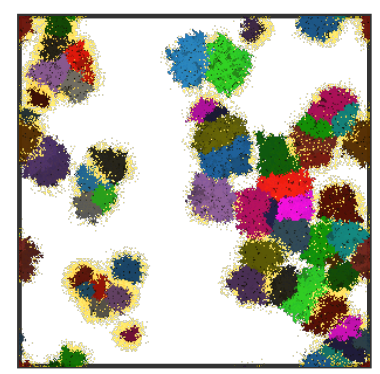

50

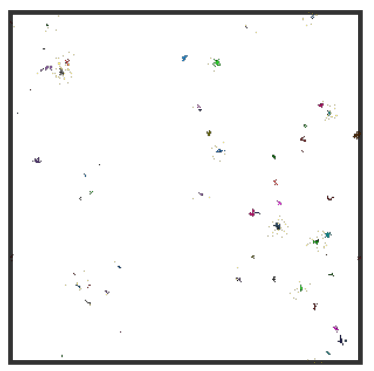

1000

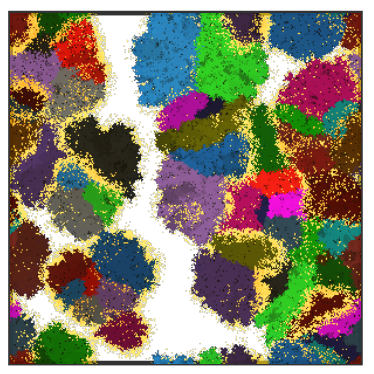

100

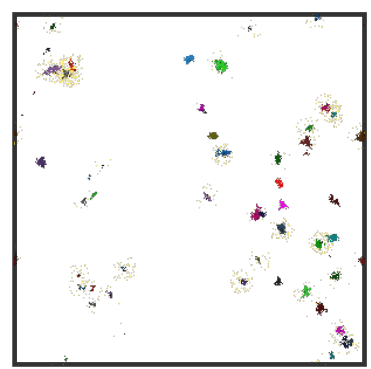

2000

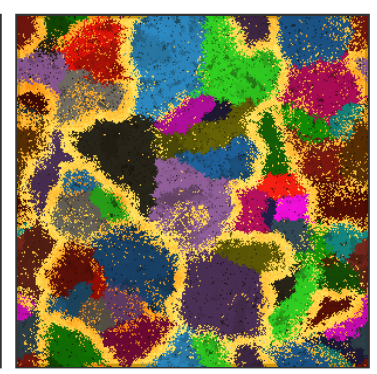

200

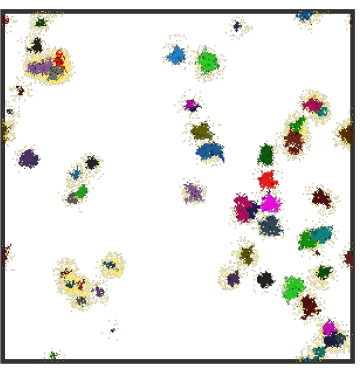

2500

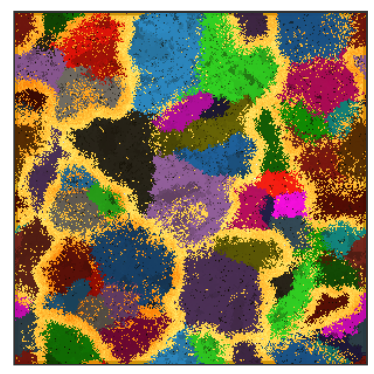

Figure SF3: The eco-evolutionary dynamics during a growth cycle. Different colors represent colonies arising from single spores. Darker shades of yellow indicate that more antibiotics are present.

\section{S3 No division of labor with weak trade-off}

Antibiotic production rate $k_{\mathrm{ab}}$ is inversely related to the number of growth-promoting genes via the expression $k_{\mathrm{ab}}=A \exp \left(-\beta_{g} g\right)$ (see Methods) - effectively imposing a trade-off between replication and antibiotic production. The strength of the trade-off is tuned by the parameter $\beta_{g}$, which in the main text is set to $\beta_{g}=1$ throughout. The closer $\beta_{g}$ is to zero, the more antibiotic production becomes independent of the number of growth genes. We expect that no division evolves with a weaker trade-off, because bacteria can both replicate and produce antibiotics. Indeed, Fig. SF4 shows that for such weaker trade-off $\left(\beta_{g}=0.5\right)$, a division of labor does not emerge within 2000 growth cycles, corresponding to $5 * 10^{6}$ time steps. 

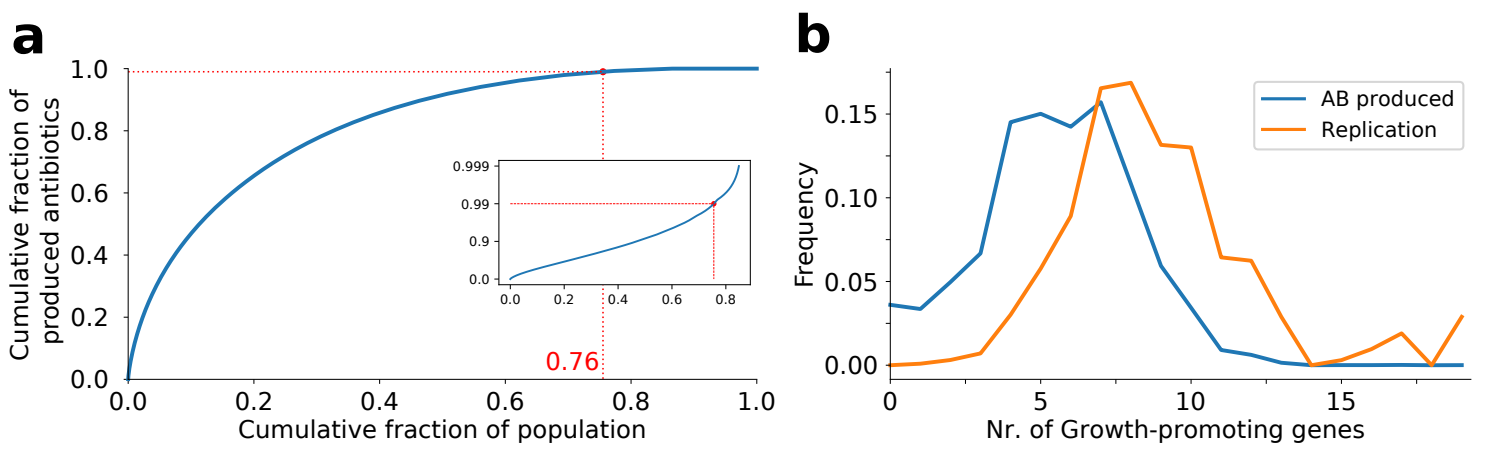

Figure SF4: No division of labor when the trade-off between replication antibiotic production is weak. Data is collected for one growth cycle after long-term evolution from a simulation with $\beta_{g}=0.5$ (all other parameters are identical to those in the caption of Fig. 1). a) Antibiotics are produced by a large fraction of the population. The cumulative fraction of the population is plotted against the cumulative fraction of all antibiotics produced. The red dotted line indicates the fraction of the population that produces $99 \%$ of the antibiotics. b) No genetic differences between antibiotic-producing and replicating individuals. Using the same data set as a), the plot shows the frequency of replication events (orange) and antibiotic production events (blue) per number of growth genes.

\section{S4 The fraction of mutants during colony development}

As shown in the main text, colonies begin their growth cycle clonally, and diversify through mutations. Mutants that overproduce antibiotics show massive deletions in their telomeres and lack growth promoting genes. We select mutants that deleted at least $3 / 4$ of their growth genes and have at least 1 antibiotic gene as a proxy for mutants that hyperproduce antibiotics (see main text Fig. 2d). Fig. SF5 shows how the fractions of these mutants changes during colony development. For colonies at an early time stage (i.e. at 40 growth cycles), mutation-driven division of labor has not evolved yet, and therefore mutations are largely deleterious and over a growth cycle mutants are outcompeted by the wild-type. At later stages, after division of labor has evolved, the fraction of mutants is much larger than for earlier cases reaching up to $7 \%$ of the population at the beginning of the growth cycle, and stabilising above $2 \%$ at the end. 


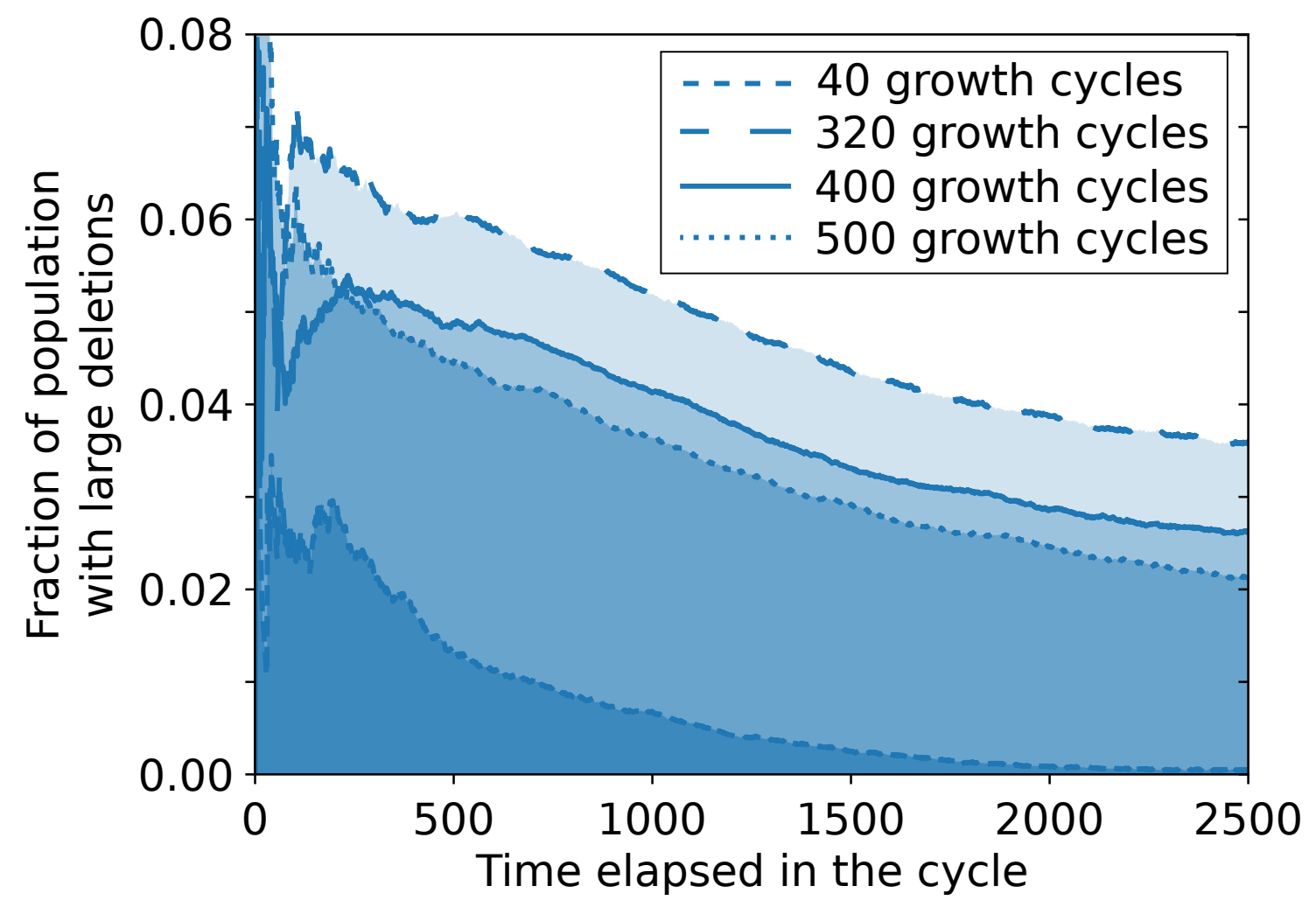

Figure SF5: The fraction of mutants during colony development (i.e. over one growth cycle of 2500 time steps), for different time points. At growth cycle 40, division of labor has not evolved yet. At growth cycle 320, 400 and 500 division of labor has evolved and mutants are stably maintained throughout the growth cycle. 


\section{${ }_{431}$ S5 Genome architecture: growth-promoting genes}

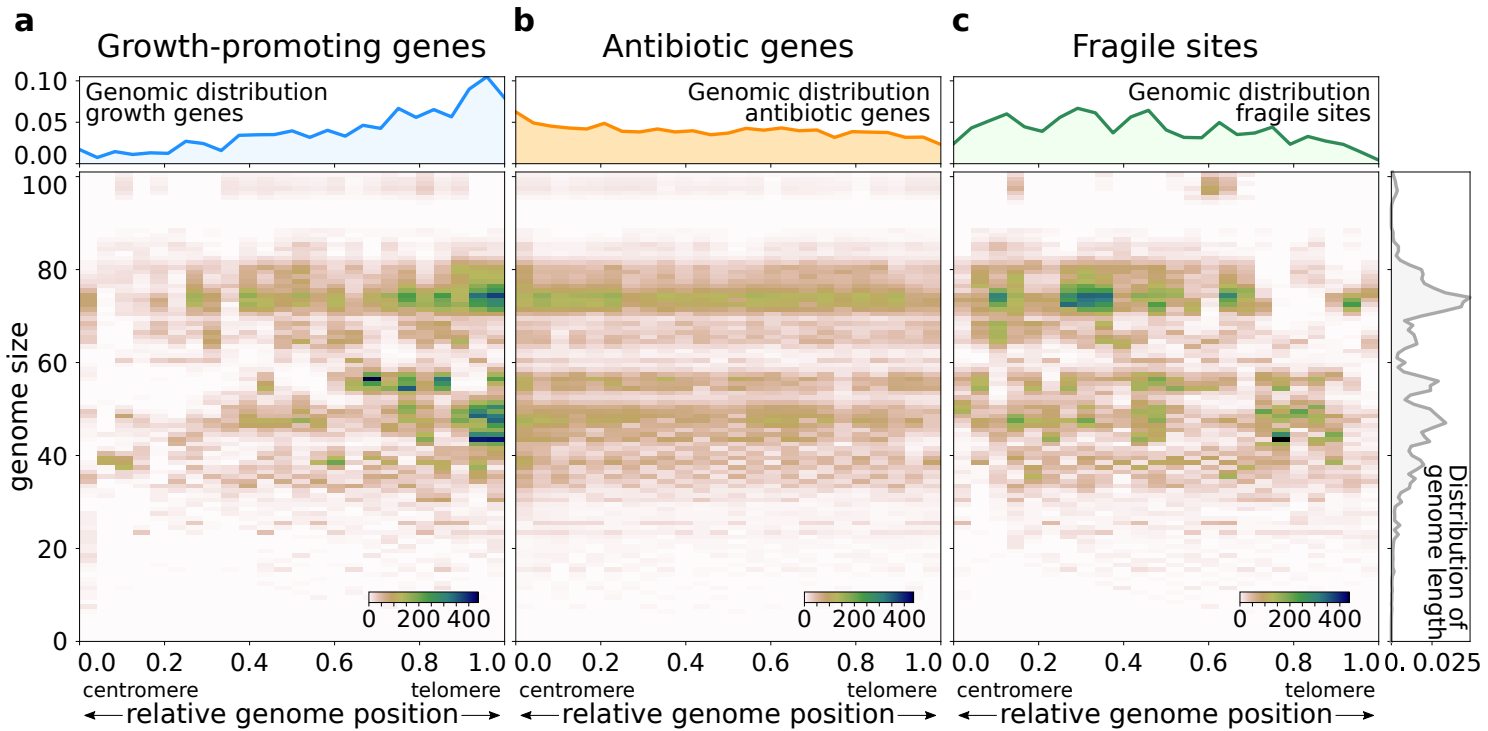

Figure SF6: The evolved genome architecture: growth promoting genes are compartmentalised to the telomeric side of fragile sites, so that fragile-site deletions growth genes in block. 


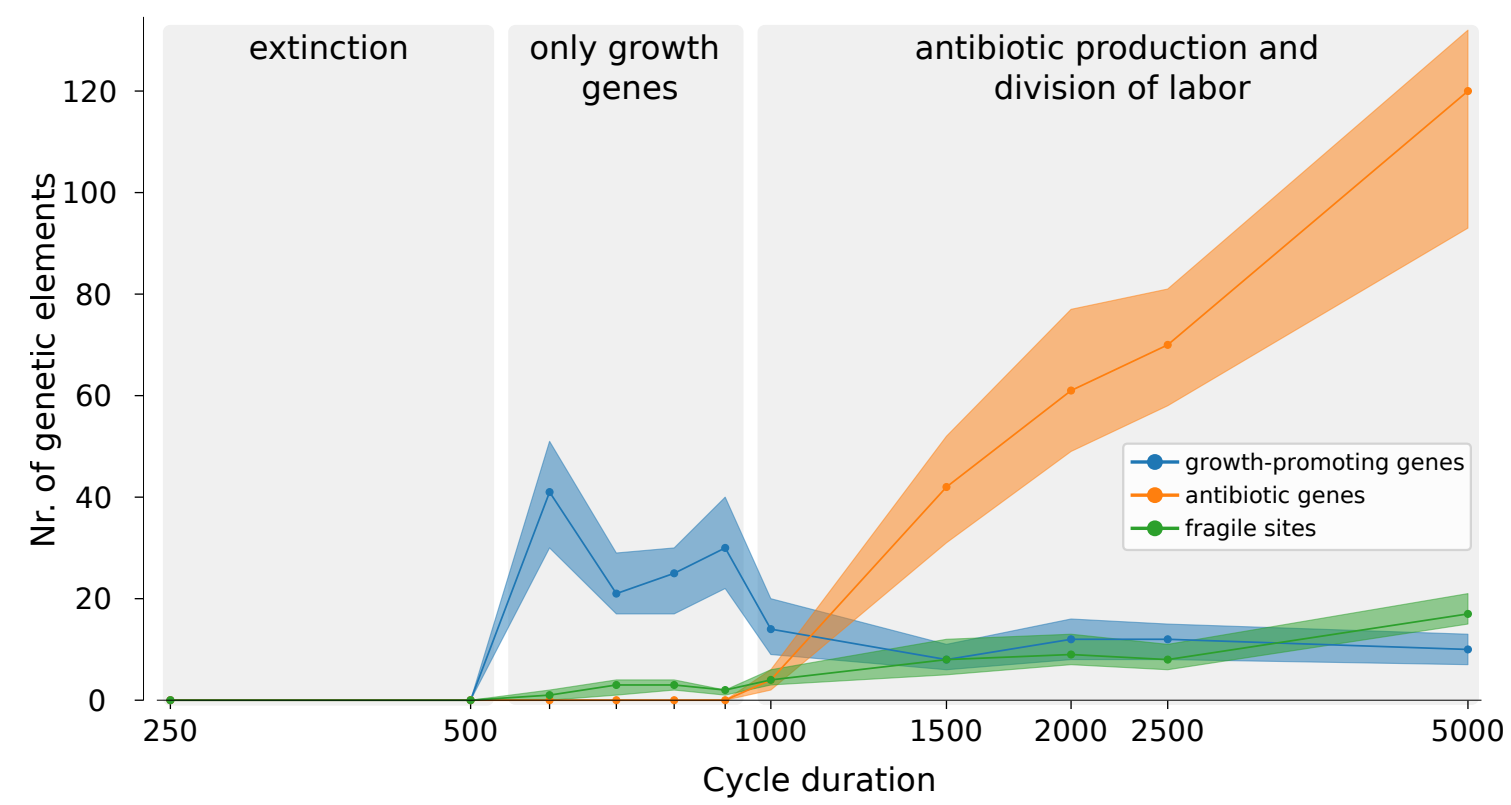

Figure SF7: The genome composition depends on the duration of the growth cycle. The plot shows the distribution of growth-promoting genes, antibiotic genes and fragile sites (after longterm evolution), for different durations of the growth cycle (for cycle duration $\leq 500$ the system goes to extinction). 


\section{S7 The effect of destroying spatial structure}

Starting from an evolved colony, we ran five simulations in which the location of bacteria was randomized at every time step. This disrupts colony formation, with two consequences: all bacteria are likely exposed to all antibiotics (in a colony this is not the case, because bacteria at its center do not come in contact with antibiotics other than those they themselves produce), and the local benefit of antibiotic production is also lost. We find that division of labor is maintained and the number of antibiotic genes increases, presumably because non-local competition always selects against loss of antibiotic resistance and favors an increase in antibiotic diversity. Fig. SF8 shows that this results in a steady increase in the number of antibiotic genes.

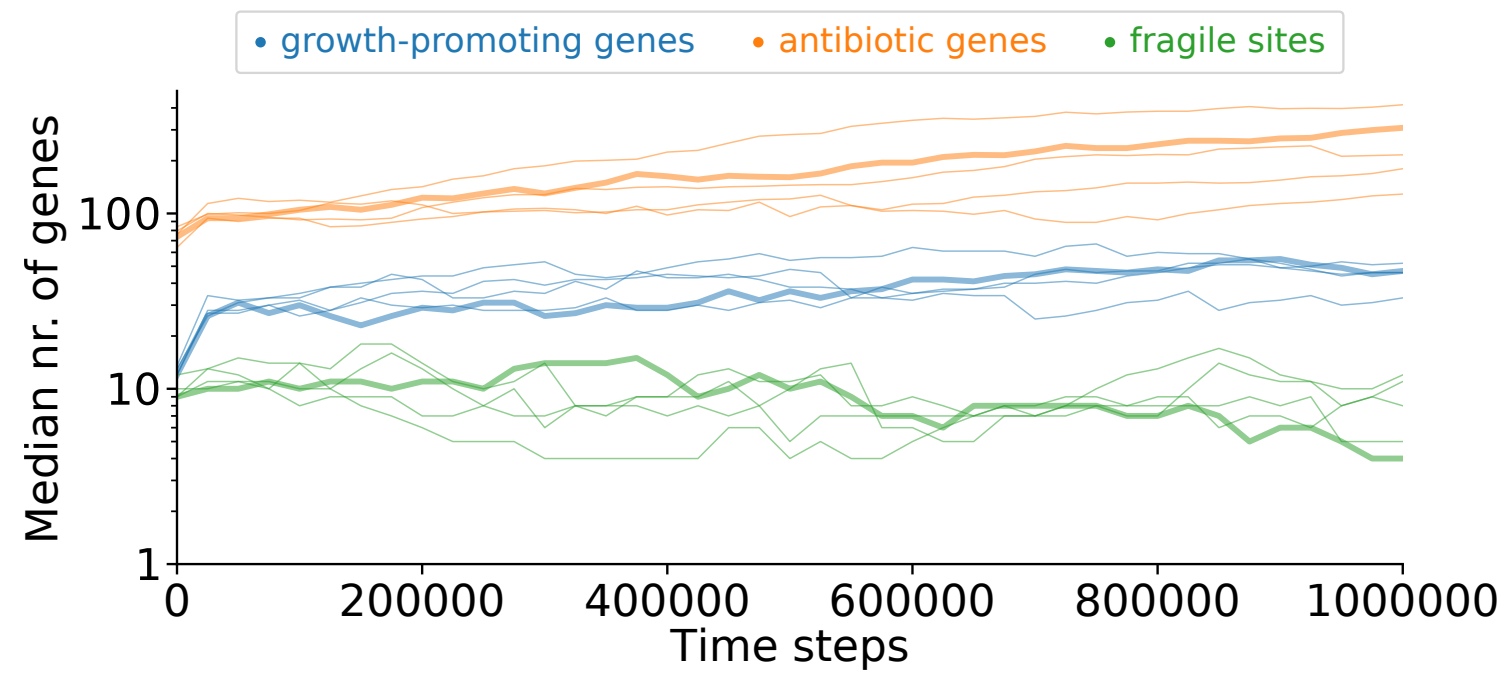

Figure SF8: Starting from genomes that divide labor, evolutionary dynamics of the genomes in the population when the system is mixed every time step. The system is initialized from evolved bacteria that divide labor. The plot shows the median number of each gene type in the genome. The lattice location of each bacterium is randomized every time step. All the runs are initialised from the same evolved colony.

We also tested the effect of disrupting spatial structure on the evolution of bacteria that do not divide labor, i.e. starting from randomly generated genomes. Fig. SF9 shows that genomes evolve a large number of growth-promoting genes, and no antibiotic genes or fragile sites, indicating that they are solely being selected on the basis of their growth rate. 


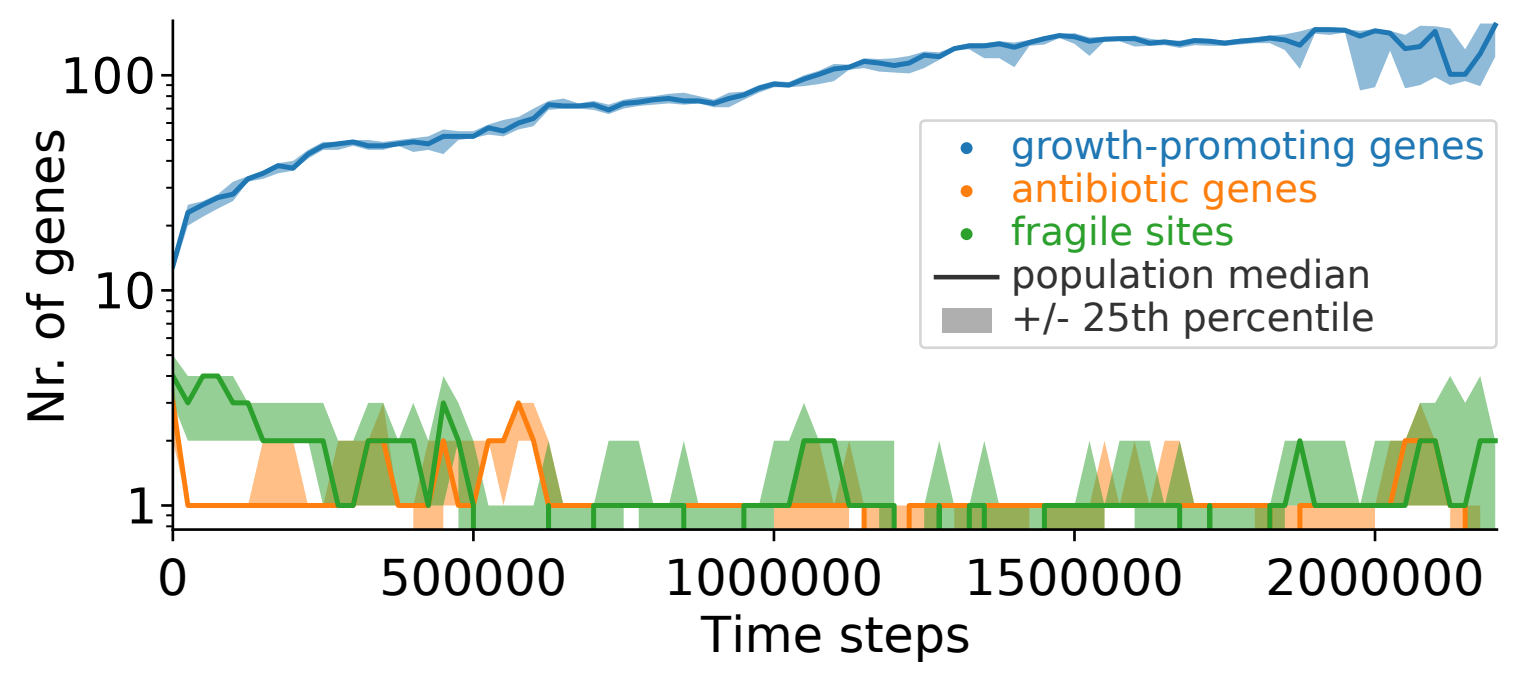

Figure SF9: Starting from random genomes, evolutionary dynamics of the genomes in the population when the system is mixed every time step. The simulation is initialised from a population of random genomes of length 20 , with average proportion of growthpromoting genes, antibiotic genes and fragile sites 12:4:4.

\section{S8 The number of antibiotic genes is due to selection for diversity}

To check that antibiotic diversity is under selection rather than the number of antibiotic genes, we modified the antibiotic production rate so that it is independent of the number of antibiotic genes. In the modified system (cf. Methods), antibiotic production per unit time $k_{\mathrm{ab} \text { production }}^{\prime}$ depends solely on the number of growth promoting genes - if at least one antibiotic gene is present, and is zero otherwise:

$$
k_{\mathrm{ab} \text { production }}^{\prime}=A^{\prime}(a) I(g)
$$

with

$$
A^{\prime}(a)= \begin{cases}0 & a=0 \\ \alpha_{a}^{\prime} & a \geq 1\end{cases}
$$

and $I(g)=\exp \left(-\beta_{g} g\right)$ as in the Methods. Fig. SF10 shows that results are robust to this change, and a large number of antibiotic genes is incorporated in the evolved genomes, indicating that selection is on antibiotic diversity rather than antibiotic number. (cf. Fig. SF2). 


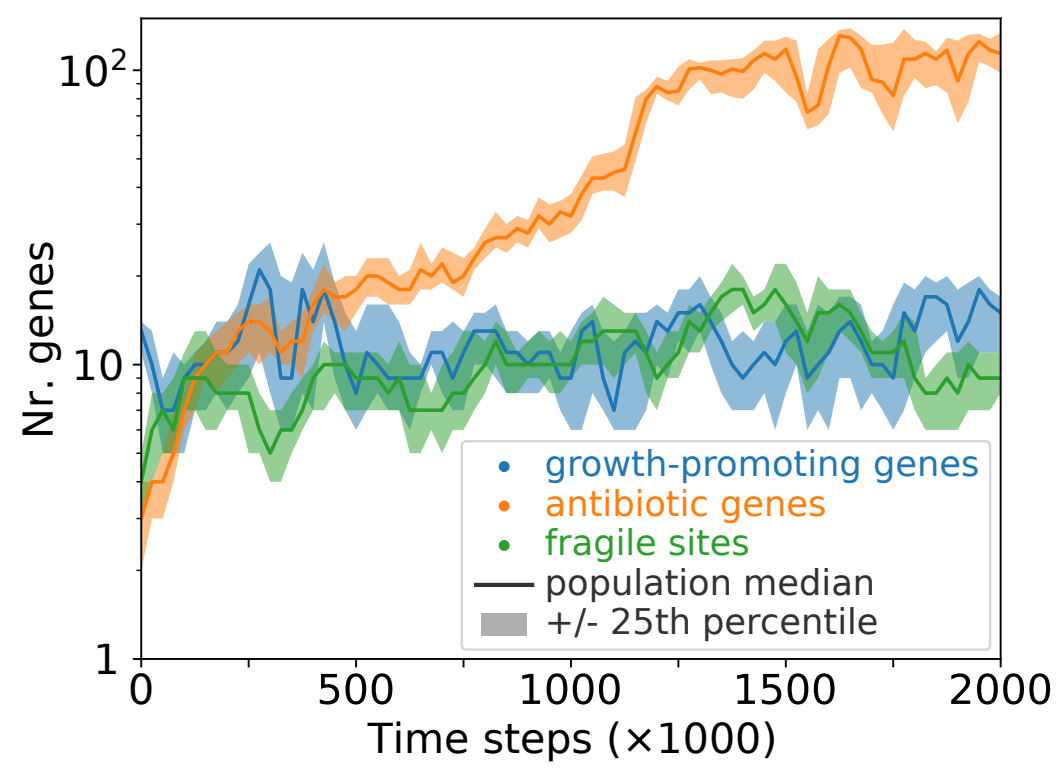

Figure SF10: A large number of antibiotic genes is incorporated in the genome despite the antibiotic production rate being independent of the number of antibiotic genes. All other parameters are identical to those of the simulation shown in Fig. SF2.

\section{S9 High and diverse antibiotic production}

The large number of antibiotic genes and their variability are due to selection for antibiotic diversity (multi-toxicity), see Fig. SF2. Fig. SF11 shows that colonies are susceptible to most antibiotics. 


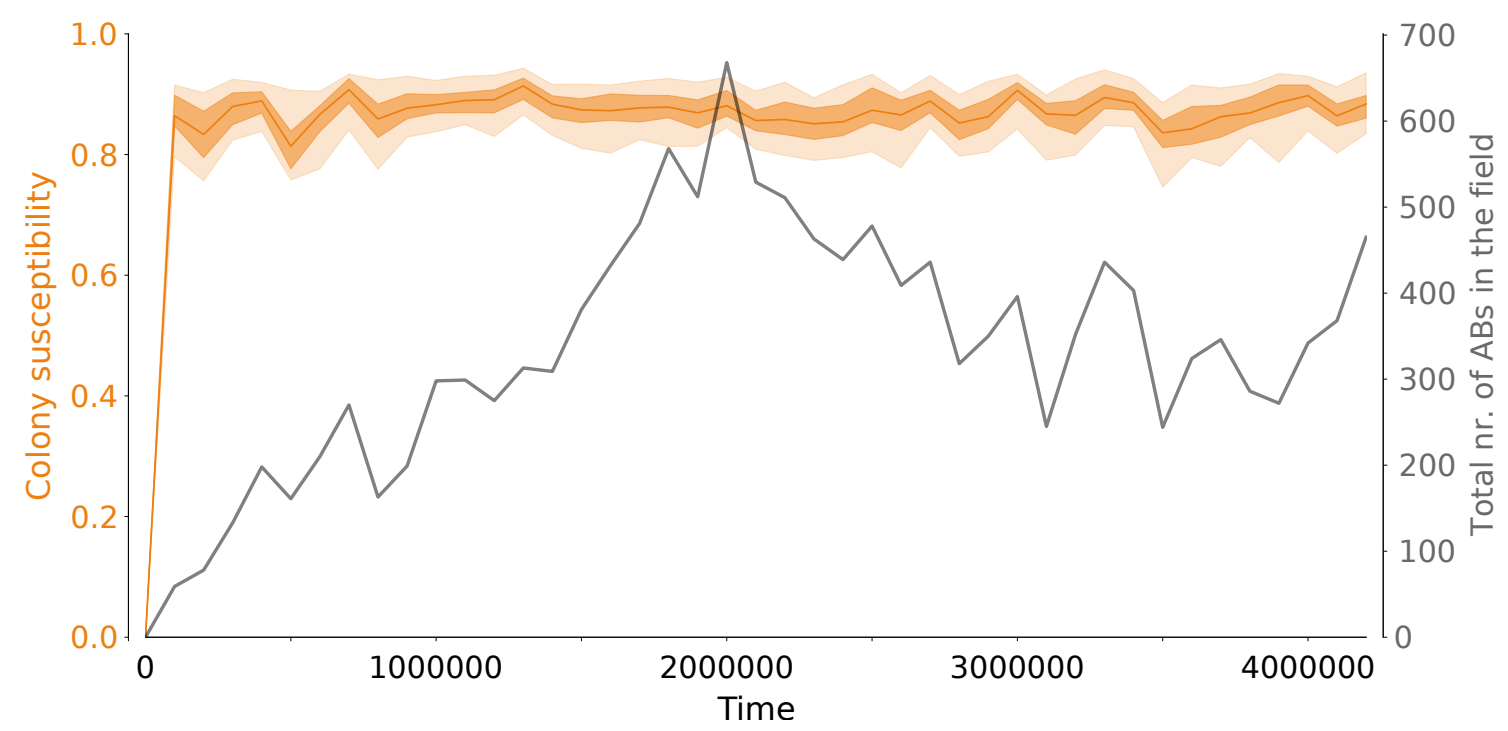

Figure SF11: Evolutionary dynamics of antibiotic production and susceptibility. We extracted the total number of antibiotics produced per time step from the same run shown in Fig. SF2, and measured the susceptibility of each bacterium in the lattice as the fraction of antibiotics that causes a $75 \%$ fitness decrease or more. Orange line: median susceptibility, shaded areas are, top to bottom, 5th, 25th, 75th and 95th percentile. The grey line shows the total number of different antibiotics in the system.

\section{S10 The total number of possible antibiotics determines the evolution of colony susceptibility}

The evolutionary potential for antibiotic diversification depends on the total number of possible antibiotics, which is determined by the length of the bit-string that defines the antibiotic (Fig. SF12; with a binary strings of length $\nu$, the volume of the antibiotic space is $2^{\nu}$ ). With long antibiotic strings $(\nu \geq 8$ ), bacteria occupy a small part of the total antibiotic space, and bacteria are susceptible to most antibiotics produced by other colonies (Fig. SF12 red line, see also Suppl. Section S9). This indicates that large antibiotic diversity promotes competition - an eco-evolutionary outcome previously named "multi-toxicity" in the context of colicin evolution models [30]). When the evolutionary potential for antibiotic diversification is small $(\nu \leq 6)$, the system reaches a different eco-evolutionary steady state - characterised by low susceptibility because each genome contains many copies of each possible antibiotic gene. This state has been previously called "hyper-immunity" and persists because the loss of resistance to any antibiotic leads to extinction. 


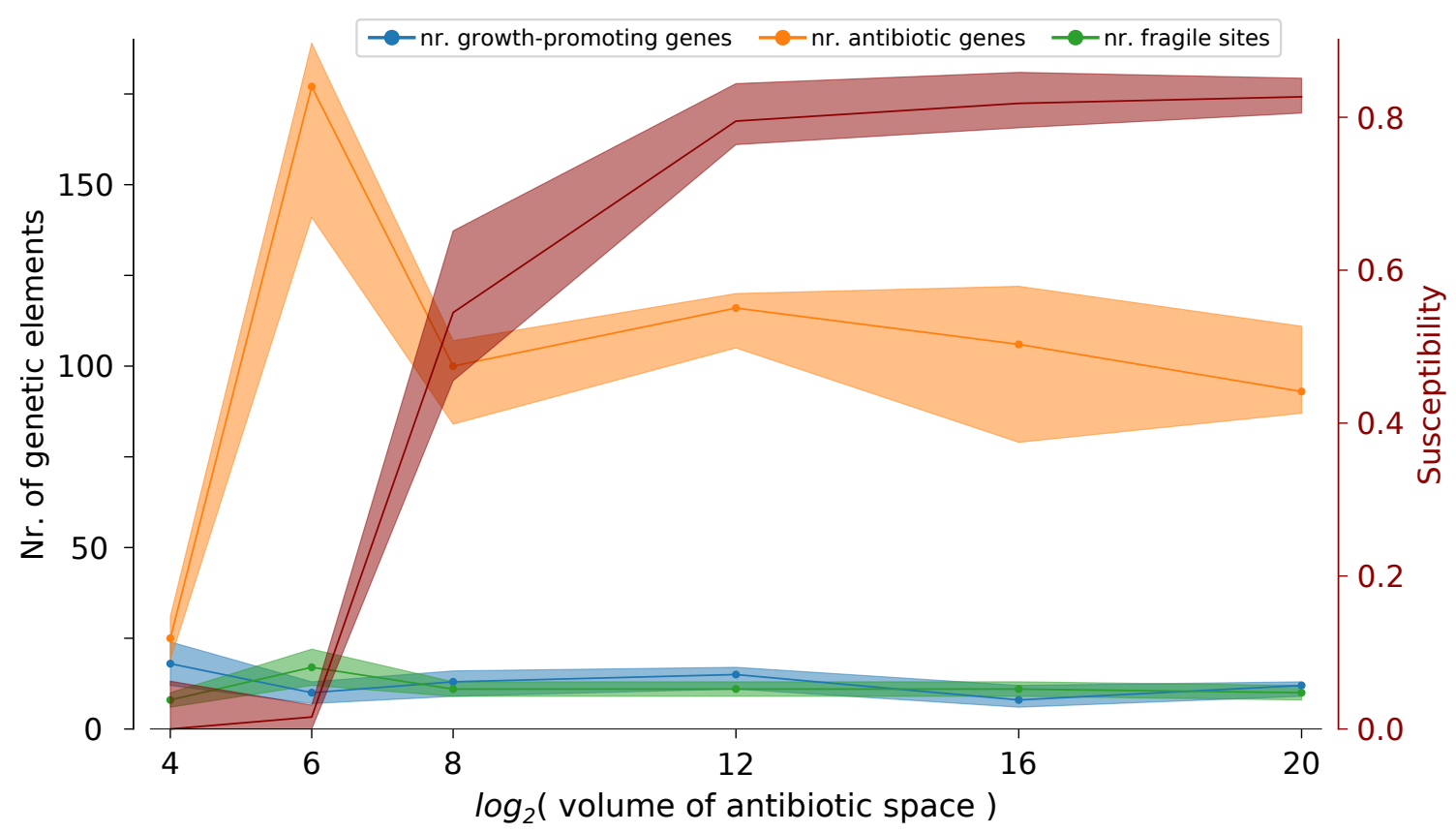

Figure SF12: The total number of possible antibiotics, i.e. the volume of the antibiotic space, determines steady-state genome size and colony immunity. We ran one simulation for each antibiotic bit-string length $\nu \in 4,6,8,12,16,20$. The volume of the antibiotic space is $2^{\nu}$. We recorded the number of genes and fragile sites after long-term evolution, as well as the colony susceptibility (defined as the fraction of all antibiotics in the filed to which the colony is susceptible).

\section{S11 The architecture of genomes evolved when antibiotic volume space is small}

We ran a simulation where the size of the antibiotic bit-string was $\nu=6$. After longterm evolution we measured the genome architecture in the population in the same way as shown in Suppl. Section S5. Fig. SF13 shows that the volume of the antibiotic space does not affect the evolution of the architecture that supports division of labor. 
bioRxiv preprint doi: https://doi.org/10.1101/2021 06.04.447040; this version posted June 4, 2021. The copyright holder for this preprint (which was not certified by peer review) is the author/funder, who has granted bioRxiv a license to display the preprint in perpetuity. It is made available under aCC-BY-NC-ND 4.0 International license.

a

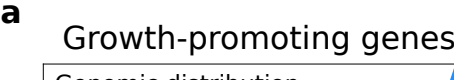

b
Antibiotic genes

$\mathbf{C}$

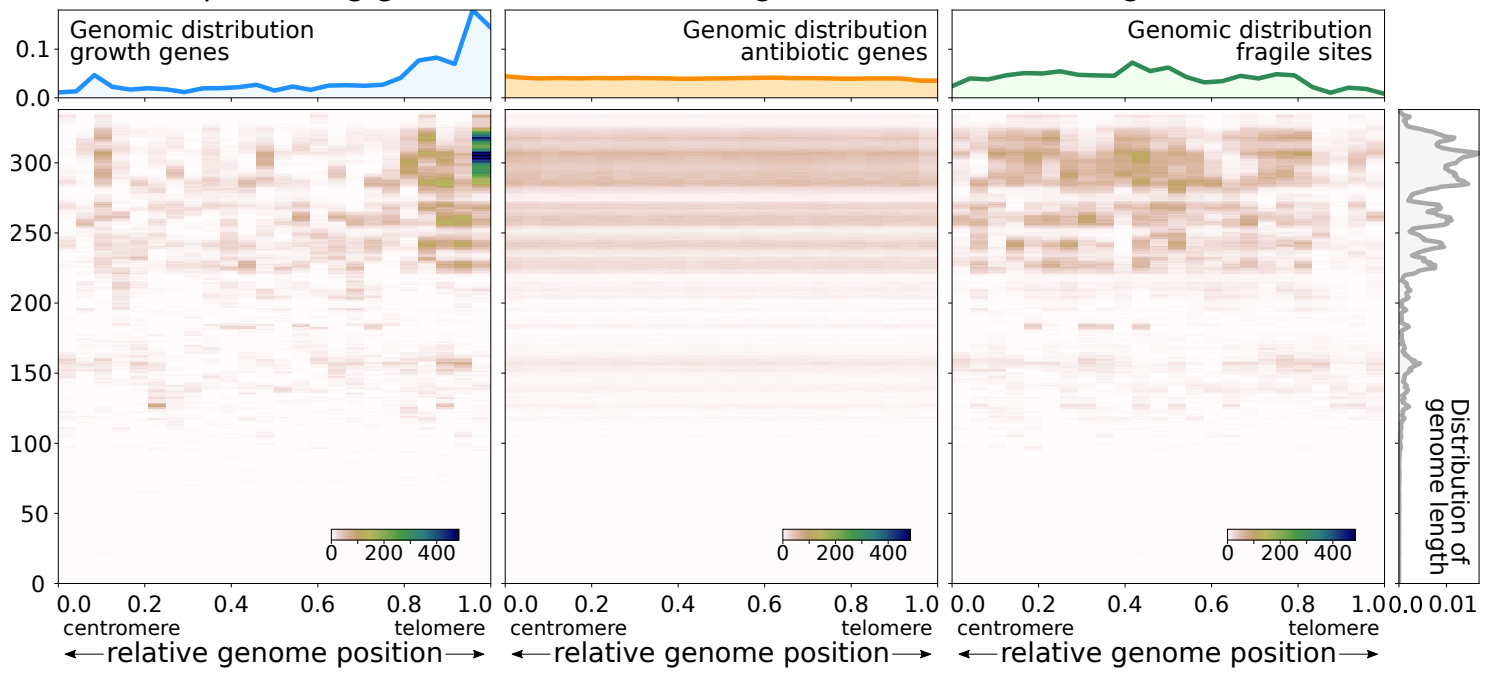

Figure SF13: Small antibiotic space $\left(2^{6}=64\right.$ different antibiotics $)$ volume does not affect division of labor. We ran a simulation identical to that shown in Fig. SF2, except for an antibiotic bit-string length of $\nu=6$. 


\section{References}

[1] William C Ratcliff, R Ford Denison, Mark Borrello, and Michael Travisano. Experimental evolution of multicellularity. Proceedings of the National Academy of Sciences, 109(5):1595-1600, 2012.

[2] Dennis Claessen, Daniel E Rozen, Oscar P Kuipers, Lotte Søgaard-Andersen, and Gilles P Van Wezel. Bacterial solutions to multicellularity: a tale of biofilms, filaments and fruiting bodies. Nature Reviews Microbiology, 12(2):115-124, 2014.

[3] Samir Giri, Silvio Waschina, Christoph Kaleta, and Christian Kost. Defining division of labor in microbial communities. Journal of molecular biology, 431(23): 4712-4731, 2019.

[4] Jordi Van Gestel, Hera Vlamakis, and Roberto Kolter. Division of labor in biofilms: the ecology of cell differentiation. Microbial Biofilms, pages 67-97, 2015.

[5] Anna Dragoš, Heiko Kiesewalter, Marivic Martin, Chih-Yu Hsu, Raimo Hartmann, Tobias Wechsler, Carsten Eriksen, Susanne Brix, Knut Drescher, Nicola StanleyWall, et al. Division of labor during biofilm matrix production. Current Biology, 28 (12):1903-1913, 2018.

[6] Enrico Sandro Colizzi and Paulien Hogeweg. Evolution of functional diversification within quasispecies. Genome biology and evolution, 6(8):1990-2007, 2014.

[7] Nobuto Takeuchi, Paulien Hogeweg, and Eugene V Koonin. On the origin of dna genomes: evolution of the division of labor between template and catalyst in model replicator systems. PLoS Comput Biol, 7(3):e1002024, 2011.

[8] Gergely Boza, András Szilágyi, Ádám Kun, Mauro Santos, and Eörs Szathmáry. Evolution of the division of labor between genes and enzymes in the rna world. PLoS Comput Biol, 10(12):e1003936, 2014.

[9] Zheren Zhang, Chao Du, Frédérique de Barsy, Michael Liem, Apostolos Liakopoulos, Gilles P van Wezel, Young H Choi, Dennis Claessen, and Daniel E Rozen. Antibiotic production in streptomyces is organized by a division of labor through terminal genomic differentiation. Science advances, 6(3):eaay5781, 2020.

[10] Iaroslav Ispolatov, Martin Ackermann, and Michael Doebeli. Division of labour and the evolution of multicellularity. Proceedings of the Royal Society B: Biological Sciences, 279(1734):1768-1776, 2011.

[11] Zheren Zhang, Bart Claushuis, Dennis Claessen, and Daniel E. Rozen. Mutational meltdown of microbial altruists in streptomyces coelicolor colonies. bioRxiv, 2020. doi: $10.1101 / 2020.10 .20 .347344$. 
[12] Carton W Chen, Chih-Hung Huang, Hsuan-Hsuan Lee, Hsiu-Hui Tsai, and Ralph Kirby. Once the circle has been broken: dynamics and evolution of streptomyces chromosomes. TRENDS in Genetics, 18(10):522-529, 2002.

[13] David A Hopwood. Soil to genomics: the streptomyces chromosome. Annu. Rev. Genet., 40:1-23, 2006.

[14] Grégory Hoff, Claire Bertrand, Emilie Piotrowski, Annabelle Thibessard, and Pierre Leblond. Genome plasticity is governed by double strand break dna repair in streptomyces. Scientific Reports, 8:5272, 12 2018. ISSN 20452322. doi: 10.1038/s41598018-23622-w.

[15] Abdoul Razak Tidjani, Cyril Bontemps, and Pierre Leblond. Telomeric and subtelomeric regions undergo rapid turnover within a streptomyces population. Scientific Reports, 10:1-10, 12 2020. ISSN 20452322. doi: 10.1038/s41598-020-63912$\mathrm{W}$.

[16] Monica I Abrudan, Fokko Smakman, Ard Jan Grimbergen, Sanne Westhoff, Eric L Miller, Gilles P Van Wezel, and Daniel E Rozen. Socially mediated induction and suppression of antibiosis during bacterial coexistence. Proceedings of the National Academy of Sciences, 112(35):11054-11059, 2015.

[17] Kalin Vetsigian, Rishi Jajoo, and Roy Kishony. Structure and evolution of streptomyces interaction networks in soil and in silico. PLoS Biol, 9(10):e1001184, 2011.

[18] Sanne Westhoff, Simon B Otto, Aram Swinkels, Bo Bode, Gilles P van Wezel, and Daniel E Rozen. Spatial structure increases the benefits of antibiotic production in streptomyces. Evolution, 74(1):179-187, 2020.

[19] Mervyn J Bibb. Regulation of secondary metabolism in streptomycetes. Current opinion in microbiology, 8(2):208-215, 2005.

[20] Gang Liu, Keith F Chater, Govind Chandra, Guoqing Niu, and Huarong Tan. Molecular regulation of antibiotic biosynthesis in streptomyces. Microbiology and molecular biology reviews: MMBR, 77(1):112, 2013.

[21] Daniel C Schlatter and Linda L Kinkel. Do tradeoffs structure antibiotic inhibition, resistance, and resource use among soil-borne streptomyces? BMC evolutionary biology, 15(1):1-11, 2015.

[22] Anton Crombach and Paulien Hogeweg. Chromosome rearrangements and the evolution of genome structuring and adaptability. Molecular biology and evolution, 24 (5):1130-1139, 2007. 
[23] Thomas Hindré, Carole Knibbe, Guillaume Beslon, and Dominique Schneider. New insights into bacterial adaptation through in vivo and in silico experimental evolution. Nature Reviews Microbiology, 10(5):352-365, 2012.

[24] Susan T Lovett. Encoded errors: mutations and rearrangements mediated by misalignment at repetitive dna sequences. Molecular microbiology, 52(5):1243-1253, 2004.

[25] Elise Darmon and David RF Leach. Bacterial genome instability. Microbiology and Molecular Biology Reviews, 78(1):1-39, 2014.

[26] Maryam Safari, Rana Amache, Elham Esmaeilishirazifard, and Tajalli Keshavarz. Microbial metabolism of quorum-sensing molecules acyl-homoserine lactones, $\gamma$ heptalactone and other lactones. Applied microbiology and biotechnology, 98(8): 3401-3412, 2014.

[27] Bartosz Bednarz, Magdalena Kotowska, and Krzysztof J Pawlik. Multi-level regulation of coelimycin synthesis in streptomyces coelicolor a3 (2). Applied microbiology and biotechnology, 103(16):6423-6434, 2019.

[28] Sampriti Mukherjee and Bonnie L Bassler. Bacterial quorum sensing in complex and dynamically changing environments. Nature Reviews Microbiology, 17(6):371$382,2019$.

[29] Tamás L Czárán, Rolf F Hoekstra, and Ludo Pagie. Chemical warfare between microbes promotes biodiversity. Proceedings of the National Academy of Sciences, 99(2):786-790, 2002.

[30] Ludo Pagie and Paulien Hogeweg. Colicin diversity: a result of eco-evolutionary dynamics. Journal of Theoretical Biology, 196(2):251-261, 1999.

[31] Bram van Dijk and Paulien Hogeweg. In silico gene-level evolution explains microbial population diversity through differential gene mobility. Genome biology and evolution, 8(1):176-188, 2016.

[32] Kalin Vetsigian. Diverse modes of eco-evolutionary dynamics in communities of antibiotic-producing microorganisms. Nature Ecology \& Evolution, 1(1):1-9, 2017.

[33] Ralph Kirby. Chromosome diversity and similarity within the Actinomycetales. FEMS Microbiology Letters, 319(1):1-10, 06 2011. ISSN 0378-1097. doi: 10.1111/j.1574-6968.2011.02242.x.

[34] Stuart A West and Guy A Cooper. Division of labour in microorganisms: an evolutionary perspective. Nature Reviews Microbiology, 14(11):716-723, 2016. 
[35] Antoine Frénoy, François Taddei, and Dusan Misevic. Second-order cooperation: Cooperative offspring as a living public good arising from second-order selection on non-cooperative individuals. Evolution, 71(7):1802-1814, 2017.

[36] Paulien Hogeweg and Ben Hesper. Evolutionary dynamics and the coding structure of sequences: multiple coding as a consequence of crossover and high mutation rates. Computers \& chemistry, 16(2):171-182, 1992.

[37] Paulien Hogeweg. Toward a theory of multilevel evolution: long-term information integration shapes the mutational landscape and enhances evolvability. pages 195224, 2012.

[38] Enrico Sandro Colizzi and Paulien Hogeweg. Transcriptional mutagenesis prevents ribosomal dna deterioration: The role of duplications and deletions. Genome biology and evolution, 11(11):3207-3217, 2019.

[39] Bram van Dijk, Frederic Bertels, Lianne Stolk, Nobuto Takeuchi, and Paul B. Rainey. Transposable elements drive the evolution of genome streamlining. bioRxiv, 2021. doi: 10.1101/2021.05.29.446280.

[40] Jacob Pieter Rutten, Paulien Hogeweg, and Guillaume Beslon. Adapting the engine to the fuel: mutator populations can reduce the mutational load by reorganizing their genome structure. BMC evolutionary biology, 19(1):1-17, 2019.

[41] Rafael Sanjuán, Andrés Moya, and Santiago F Elena. The distribution of fitness effects caused by single-nucleotide substitutions in an rna virus. Proceedings of the National Academy of Sciences, 101(22):8396-8401, 2004.

[42] Adam Eyre-Walker and Peter D Keightley. The distribution of fitness effects of new mutations. Nature Reviews Genetics, 8(8):610-618, 2007.

[43] Karen S Sarkisyan, Dmitry A Bolotin, Margarita V Meer, Dinara R Usmanova, Alexander S Mishin, George V Sharonov, Dmitry N Ivankov, Nina G Bozhanova, Mikhail S Baranov, Onuralp Soylemez, et al. Local fitness landscape of the green fluorescent protein. Nature, 533(7603):397-401, 2016.

[44] Antoine Frénoy, François Taddei, and Dusan Misevic. Genetic architecture promotes the evolution and maintenance of cooperation. PLoS Comput Biol, 9(11):e1003339, 2013.

[45] Anton Crombach and Paulien Hogeweg. Evolution of evolvability in gene regulatory networks. PLoS Comput Biol, 4(7):e1000112, 2008.

[46] Jonas Stenløkke Madsen, Mette Burmølle, Lars Hestbjerg Hansen, and Søren Johannes Sørensen. The interconnection between biofilm formation and horizontal gene transfer. FEMS Immunology \& Medical Microbiology, 65(2):183-195, 2012. 
[47] Thibault Stalder, Brandon Cornwell, Jared Lacroix, Bethel Kohler, Seth Dixon, Hirokazu Yano, Ben Kerr, Larry J Forney, and Eva M Top. Evolving populations in biofilms contain more persistent plasmids. Molecular biology and evolution, 37(6): 1563-1576, 2020.

[48] Bram van Dijk, Paulien Hogeweg, Hilje M Doekes, and Nobuto Takeuchi. Slightly beneficial genes are retained by bacteria evolving dna uptake despite selfish elements. eLife, 9:e56801, 2020.

[49] Kira S Makarova, Yuri I Wolf, Jaime Iranzo, Sergey A Shmakov, Omer S Alkhnbashi, Stan JJ Brouns, Emmanuelle Charpentier, David Cheng, Daniel H Haft, Philippe Horvath, et al. Evolutionary classification of crispr-cas systems: a burst of class 2 and derived variants. Nature Reviews Microbiology, 18(2):67-83, 2020.

[50] John R Bracht, Wenwen Fang, Aaron David Goldman, Egor Dolzhenko, Elizabeth M Stein, and Laura F Landweber. Genomes on the edge: programmed genome instability in ciliates. Cell, 152(3):406-416, 2013.

[51] V Talya Yerlici and Laura F Landweber. Programmed genome rearrangements in the ciliate oxytricha. Microbiology spectrum, 2(6), 2014.

[52] Jianbin Wang and Richard E Davis. Programmed dna elimination in multicellular organisms. Current opinion in genetics \& development, 27:26-34, 2014.

[53] Martin F Flajnik and Masanori Kasahara. Origin and evolution of the adaptive immune system: genetic events and selective pressures. Nature Reviews Genetics, 11 (1):47-59, 2010.

[54] Stefanie Mak, Ye Xu, and Justin R Nodwell. The expression of antibiotic resistance genes in antibiotic-producing bacteria. Molecular microbiology, 93(3):391$402,2014$.

[55] R J. de Boer and A. D. Staritsky. 2000. http://bioinformatics.bio.uu.nl/rdb/software.html.

[56] Bryan S Der, Emerson Glassey, Bryan A Bartley, Casper Enghuus, Daniel B Goodman, D Benjamin Gordon, Christopher A Voigt, and Thomas E Gorochowski. Dnaplotlib: programmable visualization of genetic designs and associated data. ACS synthetic biology, 6(7):1115-1119, 2017.

[57] Enrico Sandro Colizzi and Renske MA Vroomans. Streptoevol: Software and scripts for studying the evolution of mutation-driven division of labor in streptomyces. 2021. URL https://github.com/escolizzi/strepto2. 